\title{
Plasma flows and magnetic field interplay during the formation of a pore ${ }^{\star}$
}

\author{
I. Ermolli ${ }^{1}$, A. Cristaldi ${ }^{1}$, F. Giorgi ${ }^{1}$, F. Giannattasio ${ }^{1, \star \star}$, M. Stangalini ${ }^{1}$, P. Romano ${ }^{2}$, A. Tritschler ${ }^{3}$, and F. Zuccarello ${ }^{4}$ \\ 1 INAF Istituto Nazionale di Astrofisica - Osservatorio Astronomico di Roma, via Frascati 33, 00040 Monte Porzio Catone, Italy \\ e-mail: ermolli@oaroma.inaf.it \\ 2 INAF Istituto Nazionale di Astrofisica - Osservatorio Astrofisico di Catania, via S. Sofia 78, 95123 Catania, Italy \\ 3 NSO National Solar Observatory, Sacramento Peak Box 62, Sunspot, NM 88349, USA \\ ${ }^{4}$ Dipartimento di Fisica e Astronomia - Sezione Astrofisica, Università di Catania, via S. Sofia 78, 95123 Catania, Italy \\ Received 20 March 2015 / Accepted 22 December 2016
}

\begin{abstract}
Aims. Recent simulations of solar magneto-convection have offered new levels of understanding of the interplay between plasma motions and magnetic fields in evolving active regions. We aim at verifying some aspects of the formation of magnetic regions derived from recent numerical studies in observational data.

Methods. We studied the formation of a pore in the active region (AR) NOAA 11462. We analysed data obtained with the Interferometric Bidimensional Spectrometer (IBIS) at the Dunn Solar Telescope on April 17, 2012, consisting of full Stokes measurements of the Fe I $617.3 \mathrm{~nm}$ lines. Furthermore, we analysed SDO/HMI observations in the continuum and vector magnetograms derived from the Fe I $617.3 \mathrm{~nm}$ line data taken from April 15 to 19, 2012. We estimated the magnetic field strength and vector components and the line-of-sight (LOS) and horizontal motions in the photospheric region hosting the pore formation. We discuss our results in light of other observational studies and recent advances of numerical simulations.

Results. The pore formation occurs in less than $1 \mathrm{~h}$ in the leading region of the AR. We observe that the evolution of the flux patch in the leading part of the AR is faster $(<12 \mathrm{~h})$ than the evolution $(20-30 \mathrm{~h})$ of the more diffuse and smaller scale flux patches in the trailing region. During the pore formation, the ratio between magnetic and dark area decreases from 5 to 2 . We observe strong downflows at the forming pore boundary and diverging proper motions of plasma in the vicinity of the evolving feature that are directed towards the forming pore. The average values and trends of the various quantities estimated in the AR are in agreement with results of former observational studies of steady pores and with their modelled counterparts, as seen in recent numerical simulations of a rising-tube process. The agreement with the outcomes of the numerical studies holds for both the signatures of the flux emergence process (e.g. appearance of small-scale mixed polarity patterns and elongated granules) and the evolution of the region. The processes driving the formation of the pore are identified with the emergence of a magnetic flux concentration and the subsequent reorganization of the emerged flux, by the combined effect of velocity and magnetic field, in and around the evolving structure.
\end{abstract}

Key words. Sun: activity - Sun: photosphere - sunspots - techniques: high angular resolution

\section{Introduction}

Sunspots represent the best-known manifestation of solar magnetism (for a review see e.g. Solanki 2003; Rempel \& Schlichenmaier 2011). The structure and dynamics of sunspots have been investigated for a long time, but their evolution after emergence in the solar atmosphere cannot be predicted by current knowledge as yet. In addition to advance science, the ability to predict the evolution of sunspots presently entails economic and ethical consequences, by allowing efficient protection of technological systems and human life from space weather events (Hapgood 2012).

Pores constitute the first stage of the evolution of sunspots from which they mainly differ in size, and in strength and orientation of the magnetic field. High-resolution observations of isolated pores in the photosphere (e.g. Sainz Dalda et al. 2012; Sobotka et al. 2012; Guglielmino \& Zuccarello 2011; Giordano et al. 2008, and references therein) and chromosphere

\footnotetext{
$\star$ Movies associated to Figs. 1 and 4 are available at http://www . aanda.org

$\star \star$ Current address: INAF Istituto Nazionale di Astrofisica - Istituto di Astrofisica e Planetologia Spaziali, via del Fosso del Cavaliere, 100, 00133 Roma, Italy.
}

(e.g. Sobotka et al. 2013), and the study of samples of pores (e.g. Cho et al. 2010, 2013; Vargas Domínguez et al. 2010; Toriumi et al. 2014), show that these features are small sunspots without penumbra and with a prevailing vertical magnetic field that can reach up to $1-2 \mathrm{kG}$ strength in the photosphere (e.g. Sobotka et al. 2012). The periphery of pores displays strong downflows with plasma velocities that decrease with the atmospheric height; supersonic flows were also reported in the chromosphere (e.g. Lagg et al. 2007; Sobotka et al. 2012; Cho et al. 2013; Sobotka et al. 2013, and references therein). Converging horizontal motions appear around pores with velocities twice as high as those found inside them and the highest values near the border of the pores (e.g. Vargas Domínguez et al. 2012; Verma \& Denker 2014, and references therein). Like sunspots, pores host fine bright features and several types of waves (e.g. Balthasar et al. 2000; Bogdan \& Judge 2006; Stangalini et al. 2011, 2012).

High-resolution observations show that pores are formed by the merging of small magnetic elements dragged together by plasma motions (e.g. Sobotka 2003; Yang et al. 2003, and references therein). However, until recent times, limited diagnostic capabilities have impeded the understanding of whether the above process results from emergence through the photosphere 
of a magnetic field generated by global dynamo mechanisms in the solar interior, or from amplification and structuring of a magnetic field generated by local convective dynamo due to plasma motions. Current spectro-polarimetric instruments and methods, and recent numerical simulations promise to offer new levels of understanding of the processes involved in the formation of pores and larger scale magnetic structures; see for example the observational studies by Schlichenmaier et al. (2010), Rezaei et al. (2012), Bello González et al. (2012), Romano et al. (2013, 2014), and Watanabe et al. (2014) concerning the conditions that lead to the formation of penumbral regions, and the three-dimensional (3D) radiative magneto-hydro-dynamic (MHD) simulations of flux emergence by Rempel \& Cheung (2014).

In this framework, we hereby study the evolution of a magnetic region that led a diffuse dipolar flux patch to form a pore, and, at a later stage, dipolar sunspot groups in AR 11462. We analyse a unique ground-based spectro-polarimetric data set acquired during the pore formation, and complement these observations with space-borne data from the SDO mission. We focus on the photospheric processes related to the pore formation by investigating the physical conditions in the whole evolving region as observed on timescales longer than about $10 \mathrm{~min}$. The data and methods employed are presented in Sect. 2, while the results obtained are described in Sect. 3. We discuss our findings in light of other observational studies and the minute outcomes of recent simulations, and then draw our conclusions in Sect. 4.

\section{Data and methods}

\subsection{Observations}

The region analysed in our study was observed with the Interferometric Bidimensional Spectrometer (IBIS; Cavallini 2006) at the Dunn Solar Telescope of the National Solar Observatory (NSO/DST) on April 17, 2012, from 13:58 UT to 20:43 UT, within the AR NOAA 11462 (hereafter referred to as AR), at initial disk position [S24, E0.3]. The observations were assisted by the adaptive optics system of the NSO/DST (Rimmele et al. 2004), under fair conditions of atmospheric seeing. No data were acquired between 16:30 UT and 18:30 UT because of a worsening of the seeing started at about 16:15 UT.

The IBIS data consist of 223 sequences, each containing narrowband filtergrams derived from a 24-, 30-, and 25-point scan of the Fe I $617.3 \mathrm{~nm}, \mathrm{Fe}$ I $630.2 \mathrm{~nm}$, and Ca II $854.2 \mathrm{~nm}$ lines, respectively, over a field of view (FOV) of $\approx 40 \times 90 \mathrm{arcsec}^{2}$ with a cadence of $67 \mathrm{~s}$. The Fe I data include sequential measurements of the six polarization states $[I+Q, I+V, I-Q, I-V$, $I-U, I+U]$ at each wavelength position of the line sampling (FWHM 2 pm, step $2 \mathrm{pm}$ ), while the Ca II data (FWHM $4.4 \mathrm{pm}$, step $4.4 \mathrm{pm}$ ) include only Stokes $I$ measurements. Each measurement consists of a single filtergram taken with an integration time of $60 \mathrm{~ms}$ and pixel scale of $\approx 0.09$ arcsec. The above data are complemented with simultaneous broadband filtergrams obtained at $633.32 \pm 5 \mathrm{~nm}$ with the same exposure time and FOV of the narrowband data for the post-facto image restoration.

In this study we focus on the available Fe I $617.3 \mathrm{~nm}$ line data; according to Norton et al. (2006), the excitation potential, effective Landé factor, and average line-formation height of the Fe I $617.3 \mathrm{~nm}$ line are $2.22 \mathrm{eV}, 2.5$, and 250-350 km, respectively.

The AR evolution was also studied by analysing the data obtained with the Helioseismic and Magnetic Imager (HMI; Scherrer et al. 2012; Schou et al. 2012) aboard the Solar
Dynamics Observatory (SDO; Pesnell et al. 2012). In particular, we analysed the Level 1.5 SDO/HMI photospheric fulldisk filtergrams and magnetograms at the Fe I $617.3 \mathrm{~nm}$ line taken from April 15 to April 19, 2012, when the AR longitudinal distance was within $\pm 30^{\circ}$ of the central meridian. The data consist of $\approx 500$ images, each $4096 \times 4096$ pixels, with pixel size of 0.505 arcsec and cadence of 720 s. Additional information about the AR was derived from the SDO/HMI Spaceweather Active Region Patches (SHARP; Hoeksema et al. 2014; Bobra et al. 2014) maps obtained during the same time interval of the other SDO data.

\subsection{Methods}

The IBIS observations were processed with the standard reduction pipeline ${ }^{1}$ to compensate data for the dark and flatfield response of the CCD devices, instrumental blueshift, and instrument- and telescope-induced polarizations. Besides, they were also restored for seeing-induced degradations, using the Multi-Frame Blind Deconvolution technique (MFBD; van Noort et al. 2005, and references therein).

To get quantitative estimates of the physical parameters in the observed region, we performed spectro-polarimetric inversions of a subset of the IBIS measurements with the SIR code (Ruiz Cobo \& del Toro Iniesta 1992; Bellot Rubio 2003). We selected 20 time intervals during the IBIS observations that track the evolution of the region under good and stable seeing conditions. We processed, with the SIR code, a sub-array of $360 \times 350$ pixels extracted from the selected data and centred on the evolving feature.

Following common approaches (see e.g. Asensio Ramos et al. 2012; Requerey et al. 2015; Buehler et al. 2016, and references therein), we performed the data inversion by considering one component plus a stray-light component of unspecified amplitude. This latter component thus acts as a free parameter. Depending on the amount of polarization in each pixel, we considered the first component to be magnetic or quiet. We defined the magnetized pixels as those in which the total circular polarization signal is $\geq 2$ times the standard deviation of the signal estimated over the sub-array. Examples of the identified regions are given in Fig. A.1. We considered the Harvard-Smithsonian Reference Atmosphere (HSRA; Gingerich et al. 1971) as an initial guess model for the quiet regions and the same model, but modified with an initial value for the magnetic field strength of $0.2 \mathrm{kG}$, for the magnetized regions. We performed the data inversion by applying two computational cycles, each one including up to 30 iterations. At the first cycle, we considered all modelled quantities to be constant along the LOS and assigned them one node. At the second cycle, we added one node in the temperature. The magnetic field strength, inclination, azimuth, LOS velocity, and micro-turbulent velocity, however, were considered to be constant with height, i.e. the temperature was assigned two nodes, and the other quantities were given one node. The two nodes were set at $\log (\tau)=1.4$ and $\log (\tau)=-4$. Since we performed one-component inversions, the magnetic filling factor is equal to unity; we set the macroturbulent velocity to $0.75 \mathrm{~km} \mathrm{~s}^{-1}$. For quiet Sun region pixels, we gave the $I$ measurements four times the statistical weight of the $Q, U, V$ profiles; for magnetized region pixels, we did the opposite. At each iteration, the synthetic profiles derived from the solution of the radiative transfer equation were also convolved with the spectral response function of IBIS (Reardon \& Cavallini 2008) and weighted by considering

http://nsosp.nso.edu/dst-pipelines 
the stray-light contamination on the data. We estimated the latter quantity by averaging Stokes $I$ spectra in a region characterized by low polarization degree over the inverted sub-array, as in for example Bellot Rubio et al. (2000).

We tested the accuracy of the results obtained for different initializations of the inversions and chose the initialization that produced the best fit between the synthesized profile and measurement over each pixel and the largest physical consistency of the estimated quantities over the whole FOV inverted. We found that increasing the number of inversion cycles did not further improve the result of our calculation; on average less than 1215 iterations allowed the computational convergence. Examples of the results obtained and comparisons between the observed and inverted profiles at 20 positions on the analysed sub-FOVs are given in Figs. A.1-A.8. Values of the stray-light fraction, LOS magnetic field strength, field inclination and azimuth, and LOS velocity derived from our data analysis at the same positions of the compared profiles are listed in Tables A.1-A.7.

We then transformed the magnetic field inclination and azimuth derived from the data inversions into the local solar frame (LSF). We resolved the 180 degree ambiguity of the azimuth angle via the NPFC code (Georgoulis 2005).

In order to describe the horizontal proper motions in the IBIS FOV and estimate their velocity, $v_{\mathrm{H}}$, we applied the Fourier local correlation tracking method (FLCT; Fisher \& Welsch 2008, and references therein) to the available line-continuum data. We set the FWHM of the Gaussian tracking window to 0.5 arcsec to properly track magnetic structures with spatial scales smaller than the typical granular size; we made the temporal integration over a 13 min time interval. Finally, we computed the plasma LOS velocity, $v_{\mathrm{LOS}}$, by the Doppler shifts of line cores with respect to the average quiet Sun line centre position in the IBIS FOV. We computed the reference value for each filtergram of the analysed series. The series were previously processed with a subsonic filtering with a phase-velocity cut-off set to $5 \mathrm{~km} \mathrm{~s}^{-1}$.

We processed the time series of the SDO/HMI data according to Ermolli et al. (2014), by extracting from each image the $512 \times 512$ pixel $^{2}$ sub-array centred on the AR baricentre. Besides, we produced photospheric velocity maps of the horizontal plasma motions via the differential affine velocity estimator method for vector magnetograms (DAVE4VM; Schuck 2006) on the SDO/HMI SHARP data. In particular, following Schuck (2008), we derived persistent plasma motions by comparing magnetograms taken 24 min apart with a 5.5 arcsec FWHM apodization window.

\section{Results}

\subsection{AR evolution and pore formation}

The AR was observed on the solar disk from April 16 to April 22, 2012, when it reached the western limb. Figure 1 shows the AR as seen in the SDO/HMI observations taken at given times from April 16, 2012, 12:00 UT, to April 19, 2012, 10:48 UT. In each panel, we show the subfield of $\approx 160 \times 170 \operatorname{arcsec}^{2}$, centred on the $\mathrm{AR}$, that was analysed to describe the evolution of the magnetic field and radiative flux in the AR. We also show (Fig. 1, Col. 2) the two parts (sFOV) of the above subfield that were considered to describe the evolution of the trailing (negative) and leading (positive) polarity regions in the $\mathrm{AR}, \mathrm{sFOV}_{\mathrm{t}}$ and $\mathrm{sFOV}_{\mathrm{l}}$, respectively. The full temporal evolution of the analysed subfield is shown in the movie attached to Fig. 1.

According to the NOAA/USAF active region summary, in the early stages of its evolution, the AR consists of seven tiny
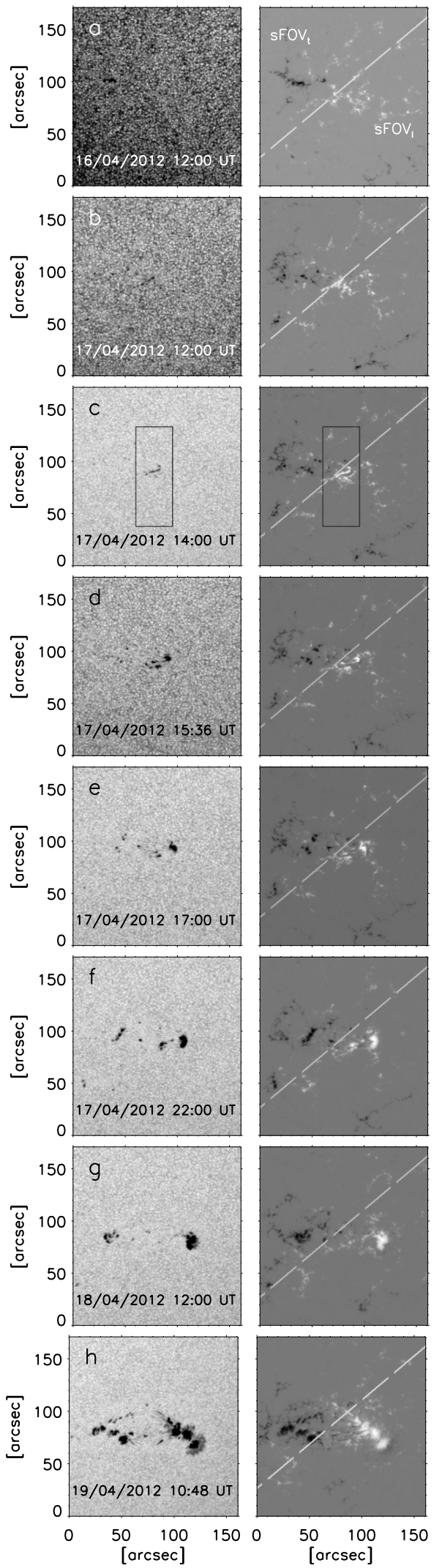

Fig. 1. AR 11462 as seen in the SDO/HMI continuum filtergrams (left) and LOS magnetograms (right) from April 16, 2012, 12:00 UT, to April 19, 2012, 10:48 UT. The box in the April 17, 2012, 14:00 UT data show the FOV of IBIS data. More details in Sect. 3.1. The magnetic field in the background magnetogram is shown in the range of values $[-1.5,1.5] \mathrm{kG}$. The full temporal evolution of the analysed data is shown in a movie available online. 


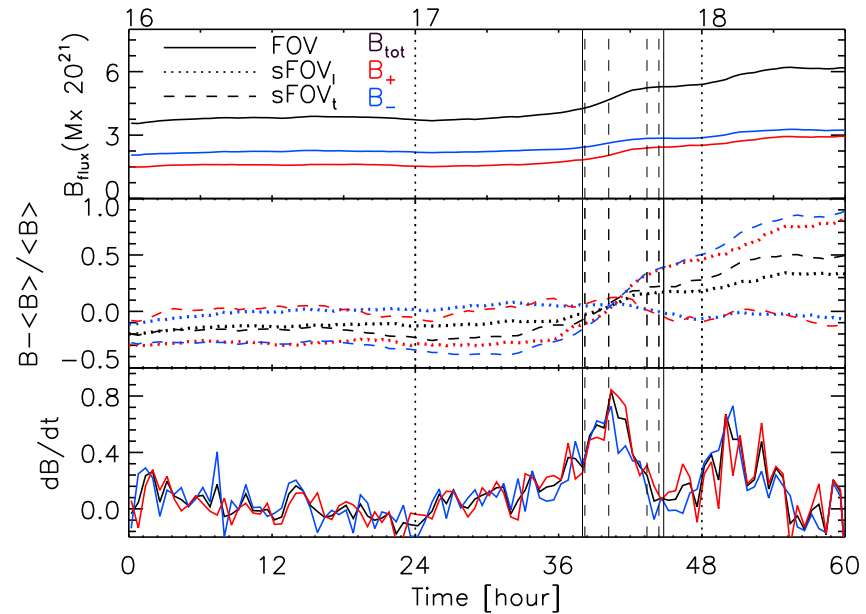

Fig. 2. Evolution of the magnetic flux (top, middle) and flux derivative (bottom) in AR 11462 from the SDO/HMI LOS magnetograms acquired from April 16, 2012, 00:00 UT, to April 18, 2012, 12:00 UT, by analysing the subfield shown in Figs. 1 (solid line), and the leading and trailing parts of the same subfield separately (dotted and dashed lines, respectively). The black, red, and blue lines indicate the total, positive, and negative magnetic flux, respectively. The vertical lines show the time interval of the IBIS observations (solid), the times the data shown in Fig. 3 were taken (dashed), and the times of 00:00 UT from April 16 to April 18, 2012 (dotted). The additional axis indicates calendar days at 00:00 UT. For clarity, flux values are only shown from data taken with a cadence of $36 \mathrm{~min}$.

field features organized to form a diffuse dipolar flux region, which is seen for example in Fig. 1 (lines $a-b)$. The outermost available observations of the AR consists of several sunspots and pores, which are already seen in the observations taken on April 19, 2012 and shown, for example in Fig. 1 (line h).

Figure 2 shows the evolution of the magnetic flux (top and middle panels) and flux derivative (bottom panel) in the AR, from the SDO/HMI LOS magnetograms taken from April 16 00:00 UT to April 18 12:00 UT, 2012, over the subfield and the two sFOVs shown in Fig. 1 and in the attached movie. The total unsigned magnetic flux $\left(B_{\text {tot }}\right)$ emerged in the AR (Fig. 2, top panel) remains almost constant with values below $4 \times 10^{21} \mathrm{Mx}$ till April 17, 2012, $\approx 12: 00$ UT, then it undergoes an increase.

The flux evolution points out an increased flux of comparable magnitude in both the $\mathrm{SFOV}_{1}$ and $\mathrm{sFOV}_{\mathrm{t}}$ on April 17, 2012, between 9:30 UT and 12:30 UT, before the start of the IBIS measurements (Fig. 2, middle and bottom panels). This flux increase, which is consistent with the flux increase by a risingtube process, precedes the formation of a filamentary, weak Sshaped structure and occurred in $\mathrm{sFOV}_{1}$ between $\approx 12: 30 \mathrm{UT}$ and 13:30 UT. The IBIS measurements targeted the evolution that leads the above filamentary structure, of positive polarity flux, to form a pore, on April 17, 2012 from 13:58 UT to 20:43 UT. Figure 1 (line c) shows the FOV of the IBIS data, whose examples are given in Fig. 3.

The time interval of the IBIS measurements is characterized by a steep increase of flux in the AR lasting until April 17, 2012, $\approx 17: 00$ UT (Fig. 2, middle and bottom panels) and small flux changes afterwards. Available observations show that on April 17, 2012 between 15:15 UT and 15:30 UT the right end of the initial S-shaped structure breaks away at roughly onethird of the length of the structure. After the break, the right end of the structure evolves to form the pore, which at some first stages resembles a tiny U-shaped (e.g. SDO/HMI observations at

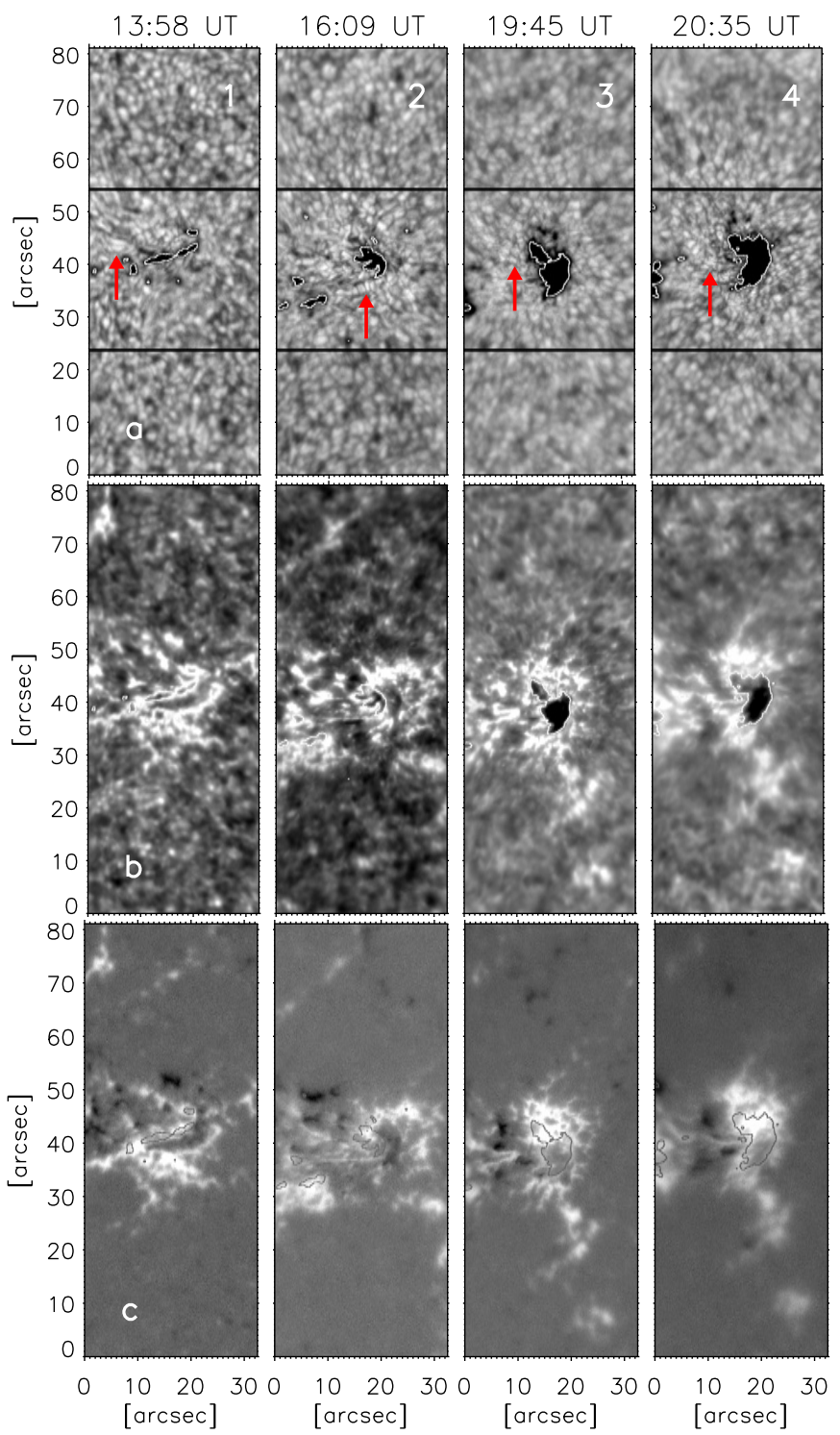

Fig. 3. Examples of the IBIS Fe I $617.3 \mathrm{~nm}$ line data analysed in our study; letters (numbers) between brackets indicate the line (column) label. a) Stokes $I$ in the continuum near the line, b) Stokes $I$ in the line core, c) Stokes $V$ in the blue wing, near the line core, at four stages of pore evolution, from data taken at (1) 13:58 UT, (2) 16:09 UT, (3) 19:45 UT, and (4) 20:35 UT. North (west) is at the top (right). The black box in the top panels shows the subfield inverted with the SIR code and shown in Figs. 5 and 6. The contours overplotted in each panel indicate the location of the evolving structure, as singled out by applying an intensity threshold criterion, $I_{\mathrm{c}}<0.9 I_{\mathrm{gs}}$, where $I_{\mathrm{qs}}$ is the average quiet Sun intensity. More details are provided in Sect. 3.1.

15:36 UT), then a trident-shaped structure (e.g. 16:09 UT, IBIS data in Fig. 3, Col. 2). In the early stages of its evolution, the S-shaped structure is aligned to about 10 degree to the east-west direction (Fig. 3, Col. 1). The IBIS observations show elongated granules near the evolving structure, mostly located east of this structure in agreement with, for instance Centeno (2012) and Verma et al. (2016); these granules are oriented almost perpendicularly to the axis of the evolving feature at the early stages of its evolution and parallel to it later on (see e.g. the IBIS observations in Fig. 3 line a, granules indicated by the red bar in each panel). 
The formation of the positive polarity pore is accompanied by the emergence of small-scale magnetic features of opposite polarity. Indeed, since the early stages of the pore formation, i.e. between 10:00 UT and 12:00 UT and more extensively after 14:00 UT, the SDO/HMI observations show a tiny magnetic patch of negative polarity flux that emerges north-east of the evolving, positive flux structure, at about 100 degree to the eastwest direction (see e.g. the movie attached to Fig. 1). This diffuse negative polarity patch mostly lies outside the FOV of the IBIS observations. It nearly preserves the same size during the pore formation, but similar sized negative flux features appear south of it (e.g. the SDO/HMI observations at 16:12 UT in the movie attached to Fig. 1). The negative polarity features show clear links to the evolving, positive polarity region. This suggests that they belong to the same emerging flux loop from which the AR evolution could be ensued. The negative patches are clearly seen on the available data at the time the pore has already increased significantly in size. Near the evolving positive flux structure, the SDO/HMI magnetograms also show small-scale, mixed polarity features that counterstream; as seen in the movie attached to Fig. 1, the negative flux moves towards the $\mathrm{sFOV}_{t}$, while the positive polarity flux moves towards the $\mathrm{sFOV}_{l}$. This arrangement of opposite polarity flux resembles the footpoints of an emerging loop. The SDO/HMI magnetograms show the growth of flux regions by coalescence of smaller scale, same polarity features. This process also shows up as short-lived, small-scale light features observed in the photospheric available observations, in both the SDO/HMI and IBIS data, near the eastward side (leftmost) of the evolving structure.

Both the SDO/HMI and IBIS observations reveal a counterclockwise rotation of the growing pore, which is clearly seen as a swirling motion of the plasma immediately outside the western (rightmost) border of the evolving structure. Figure 4 shows some maps of the horizontal motions derived from the SDO/HMI data; the full evolution of all the computed maps is shown in the movie attached to Fig. 4. In these maps, the arrows were plotted to show persistent motions ( $>12 \mathrm{~min}$ ) at moderate resolution $(>2$ arcsec). Figure 4 and the attached movie show the abovementioned rotation on larger size regions of both polarities in the AR with velocities up to about $1 \mathrm{~km} \mathrm{~s}^{-1}$ (see e.g. the maps at 16:00 UT and 19:12 UT). In addition, they also display an outwards motion of the formed pore, with respect to the primary flux patch, and a drift of the negative polarity flux in the opposite direction (see e.g. the maps at 19:12 UT and 20:48 UT). As seen on the movies available online, all together these plasma motions seem to foster the accumulation of flux. Figure 4 also shows that, after the pore formation, the average velocity of the horizontal plasma motions at the evolving region reduces to about $0.3-0.4 \mathrm{~km} \mathrm{~s}^{-1}$ (Fig. 4, bottom panel).

Throughout the duration of the IBIS observations, the coherent structure resulting from the evolution of the S-shaped feature displays a fragmented, changing core in the IBIS $617.3 \mathrm{~nm}$ data and lack of penumbra around its entire perimeter. The formed pore shows a rather symmetric funnel-shaped structure (Fig. 3, Col. 4) that lasts about 9 h. During the same time interval, the field aggregation in the trailing (negative) polarity region forms in a smaller scale $(<10$ arcsec) and more diffuse features. While the pore is formed in the leading region in less than $1 \mathrm{~h}$, the more diffuse and smaller scale magnetic structures on the trailing region show a more gradual increase in flux over a time interval of 20-30 h.

On April 18-22, 2012, the total magnetic flux estimated in the evolving region slightly increases by remaining almost constant for about $24 \mathrm{~h}$, and then it increases up to values close to
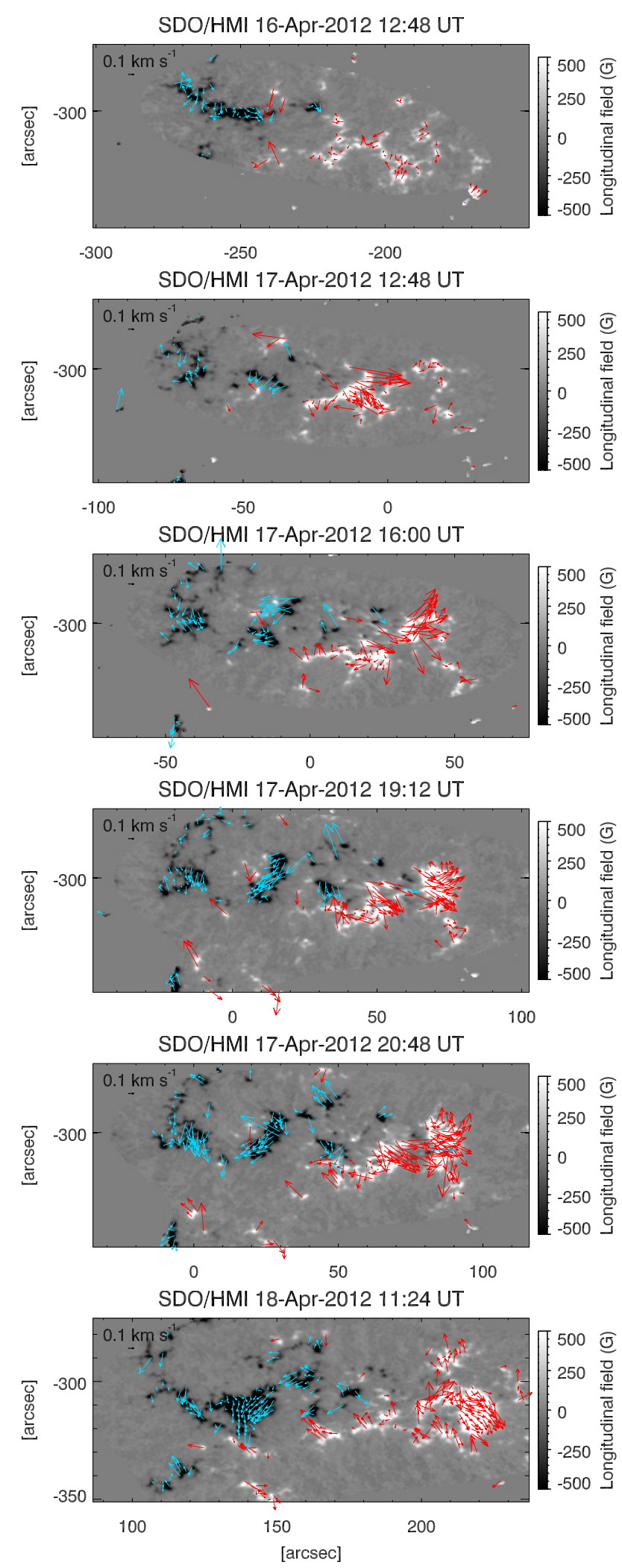

Fig. 4. Examples of the photospheric horizontal velocity maps derived from the SDO/HMI SHARP data taken from Apr. 16, 2012, 12:48 UT to Apr. 18, 2012, 11:24 UT, which is from $24 \mathrm{~h}$ before the start of the pore formation to several hour after. The arrows indicate the horizontal velocity; red (light blue) shows the leading (trailing), positive (negative) polarity. In all panels but the first, the studied pore coincides with the largest size structure in the leading region. The magnetic field in the background magnetogram is shown in the range of values specified in the colour bars. The full temporal evolution of all computed maps is shown in a movie available online. 
$9 \times 10^{21} \mathrm{Mx}$ (not shown in Fig. 2). This second flux increase follows the formation of a partial penumbra around the formed pore (Fig. 1, line g). This flux leads, in about $10 \mathrm{~h}$, to the final configuration of the region, which consists of a spot in the leading polarity region of the AR, two smaller spots in the same area, and several pores in the trailing flux region of the AR (Fig. 1, line h). The leading spot shows a partial penumbra on the side opposite from the following polarity of the AR, as reported, for example by Schlichenmaier et al. (2010). The steepest change of the total flux during the analysed time interval occurs during the formation of coherent magnetic structures eastward of the mature leading spot in the leading polarity region.

\subsection{Magnetic and velocity fields during the pore formation}

\subsubsection{Magnetic field}

Figure 5 shows examples of the results derived from the SIR inversion of the IBIS data. In particular, we show the maps of the magnetic field strength $(B)$, and of the field inclination $(\theta)$, transverse $\left(B_{\mathrm{t}}\right)$ and longitudinal $\left(B_{1}\right)$ field components in the LSF, at seven representative stages of the pore formation.

The $B$ maps (Fig. 5, Col. 2) indicate that the field extends beyond the visible outline of the evolving feature during the whole pore formation; the field reaches values up to $1-2 \mathrm{kG}$ during the entire interval analysed and slightly increases over time. At the initial stages, the most intense field concentrations are found at the edges of the evolving region; these strong fields are located near magnetic fields with lower strength and opposite polarity than those found in the evolving feature (Fig. 5 lines b, c), from which they are seen to move away (Fig. 5 lines e-g). At the initial stages, the magnetic area is $4-5$ times larger than the photometric area, as reported from analysis of an evolving small AR, for example by Verma et al. (2016), while after the formation of the coherent feature, the magnetic region is about 2 times larger than the dark structure. At the latter stages, the most intense fields are detected in the central-southern region of the pore. The field is almost vertical with respect to the photosphere in the central section of the evolving feature, and rather inclined outside it (e.g. $B_{1}$ and $B_{\mathrm{t}}$ maps in Fig. 5, Col. 4). Indeed, the $\theta$ maps (Fig. 5, Col. 3) show a large patch characterized by values of $\approx 45^{\circ}$ over an area extending well beyond that of the evolving feature, which hosts fields with $\theta<\approx 25^{\circ}$. The $\theta$ maps also show small patches of magnetic field characterized by an inclination of about $90-180^{\circ}$, which correspond to the opposite polarity features observed outside the evolving pore, north-east of it.

After the formation of the coherent, funnel-shaped feature, the $B_{\mathrm{t}}$ maps (Fig. 5, Col. 4, lines e-g) show magnetic fields that are aligned along the direction of the opposite polarity patches in the evolving region facing each other. Comparison between the maps derived from the data taken at the initial (Fig. 5, lines a-d) and final (Fig. 5, lines e-g) evolutionary stages shows the same azimuth pattern on both the fragmented positive polarity patches of the forming pore and the later formed pore. Therefore, the fields in the smaller scale, the same polarity features, and those in the formed pore are coaligned. Since the former features are seen to coalesce and form the pore, they show up as the leading footpoints of an emerging dipole.

\subsubsection{Line-of-sight and horizontal motions}

Figure 6 shows examples of the $v_{\mathrm{LOS}}$ and $v_{\mathrm{H}}$ maps derived from the IBIS data at the same stages of the pore formation presented in Fig. 5.
The $v_{\text {LOS }}$ maps (Fig. 6, Col. 1) show downflows (corresponding to positive values in the velocity maps) characterized by velocities up to $2 \mathrm{~km} \mathrm{~s}^{-1}$ in the area of the evolving structure and its periphery. These downflows occur during the whole pore formation; as the feature evolves, they become stronger, which is likely as a consequence of the flux coalescence. They are mostly located at the northern edge of the feature during the initial and intermediate evolutionary stages (Fig. 6, lines a-d), and at its eastern and southern sides during the final stages (Fig. 6, lines $\mathrm{e}-\mathrm{g})$. Upflows are suppressed in the evolving region during most of its evolution (Fig. 6, lines a-d), while they appear after the pore formation mostly at its western region (Fig. 6, line g). The maps also show convective upflows around the evolving region and localized downflows, where magnetic flux accumulates (e.g. Fig. 6, lines c, d, g, to the left side of the map). These plasma motions are also characterized by velocities up to about $1-2 \mathrm{~km} \mathrm{~s}^{-1}$. It is worth noting that the positive (negative) values of plasma velocity shown with red (blue) colours in the $v_{\text {LOS }}$ maps do not correspond to pure downflows (upflows) since they were derived from analyses of observations taken off disc centre.

The $v_{\mathrm{H}}$ maps (Fig. 6, Col. 2) show plasma motions in agreement with those inferred from the analysis of the SDO/HMI observations (Fig. 4). However, with respect to the latter maps, those in Fig. 6 more clearly represent the diverging motions of plasma seen from the visual inspection of the available data in the close proximity of the evolving region. At the initial stages of the pore formation, there are horizontal motions at both sides of the elongated evolving structure (Fig. 6, lines a-b), inwards to its upper (northern) edge and outwards beyond its lower (southern) boundary; these motions push the evolving feature forward. At these stages, the $v_{\mathrm{H}}$ maps obtained from the IBIS data also clearly show the swirling motion of the plasma in the area of the evolving feature (see e.g. Fig. 6 lines a, d). After the pore formation, the maps show coherent motions inwards directed around most of the eastward visible outline of the pore (Fig. 6, line g). After the formation of the funnel-shaped structure, the highest values in the $v_{\mathrm{H}}$ maps are found to be located far from the visible outline of the evolving structure, at a distance of about 5-10 arcsec, as reported for example by Vargas Domínguez et al. (2010).

\subsubsection{Temporal evolution}

Figure 7 shows the evolution of the physical quantities discussed above, as derived from the SIR inversion and other methods applied to the IBIS data. Each panel represents the average and standard deviation of the various quantities estimated inside and around the evolving feature.

Inside the evolving feature, $B$ varies from about $1 \mathrm{kG}$ to $2 \mathrm{kG}$, by reaching average values larger than $1.5 \mathrm{kG}$ after the formation of the funnel-shaped pore (Fig. 7, top panel). The maximum (not shown in Fig. 7) and average values of $B$ increase in time, as well as the same quantities for both the $B_{\mathrm{t}}$ and $B_{1}$ components. Outside the forming pore, the average value of $B$ ranges between $0 \mathrm{kG}$ and $0.1 \mathrm{kG}$, but there are regions in this area with magnetic field strength $\geq 1 \mathrm{kG}$. The average value of $B$ over the whole area does not change significantly during the pore formation; the same holds for both the mean value of $B$ over the stronger field elements and the maximum value of $B_{\mathrm{t}}$ in the whole area, while the maximum values of $B_{1}$ and $B$ outside the evolving pore slightly decrease $(\leq 10 \%)$ over time.

During the interval of the IBIS observations, $\theta$ does not change significantly (Fig. 7, middle panel). The average and the range of $v_{\text {LOS }}$ values measured over the magnetic region slightly decrease after the pore formation, compared to those estimated 
I. Ermolli et al.: Velocity and magnetic fields in a forming pore
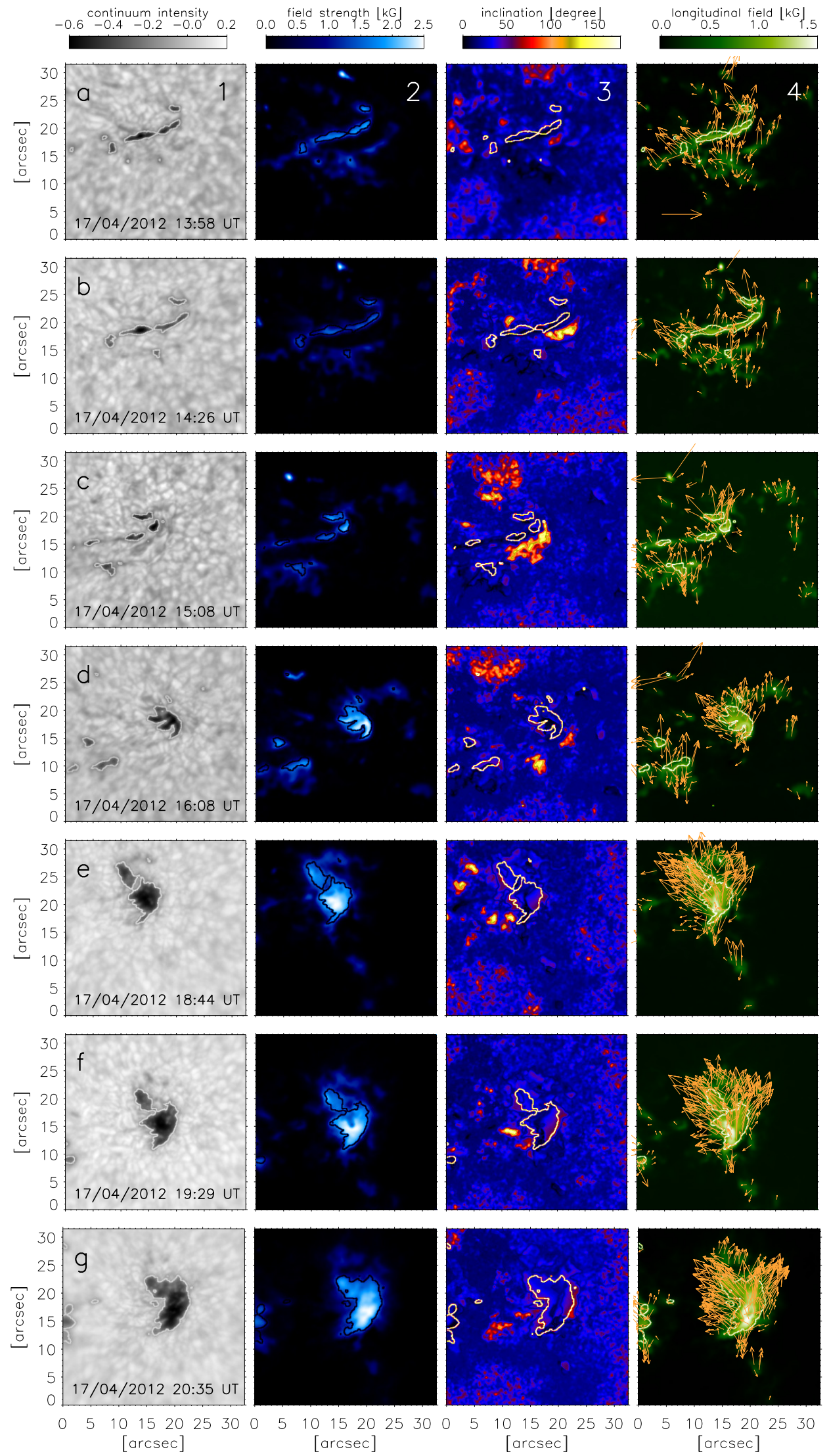

Fig. 5. From left to right, top to bottom, numbers and letters within brackets indicate the column and line labels, respectively. Examples of (1) continuum intensity, (2) magnetic field strength, (3) inclination, (4) $B_{1}$ longitudinal (background image), and $B_{\mathrm{t}}$ transverse (overplotted vector field) components of the magnetic field in the LSF derived from the SIR inversion of the IBIS Fe I $617.3 \mathrm{~nm} \operatorname{line}$ data, at optical depth $\log \tau_{500}=1$, at seven stages of the pore formation, at a) 13:58 UT, b) 14:26 UT, c) 15:08 UT, d) 16:08 UT, e) 18:44 UT, f) 19:29 UT, and g) 20:35 UT. North is at the top, and west is to the right. The arrow at the bottom left on panel 4a) represents a horizontal field of $1 \mathrm{kG}$; transverse field components lower than $0.2 \mathrm{kG}$ are not shown. The contour line in each panel shows the location of the evolving structure singled out in the continuum data, as specified in Fig. 3. 

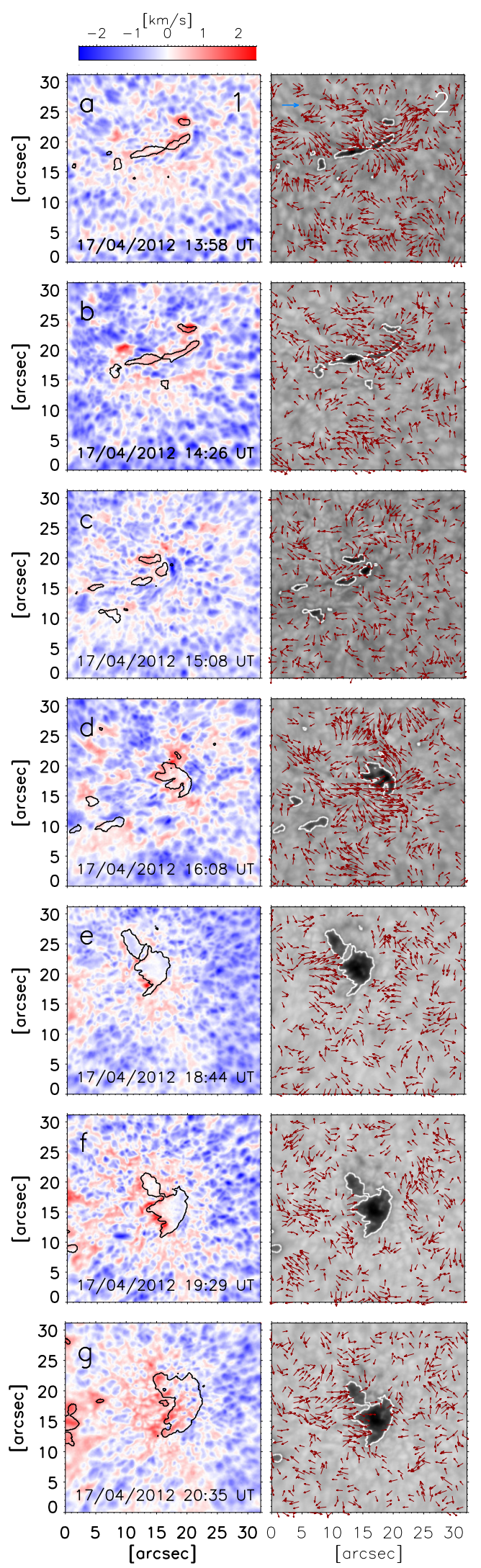

Fig. 6. Example of the $v_{\mathrm{LOS}}(\mathrm{Col} .1)$ and $v_{\mathrm{H}}$ (Col. 2) plasma velocity fields in the evolving region derived from the IBIS Fe I $617.3 \mathrm{~nm}$ data at the seven stages (lines a-g) of the pore formation shown in Fig. 5 and specified in each panel of Col. 1. The blue (negative) and red (positive) $v_{\text {LOS }}$ indicate upflows and downflows, respectively. The intensity background in the $v_{\mathrm{H}}$ maps shows the average image of the representative series. In panel $2 \mathbf{a}$, the horizontal blue bar indicates $v_{\mathrm{H}}$ plasma velocity of $1 \mathrm{~km} \mathrm{~s}^{-1}$.

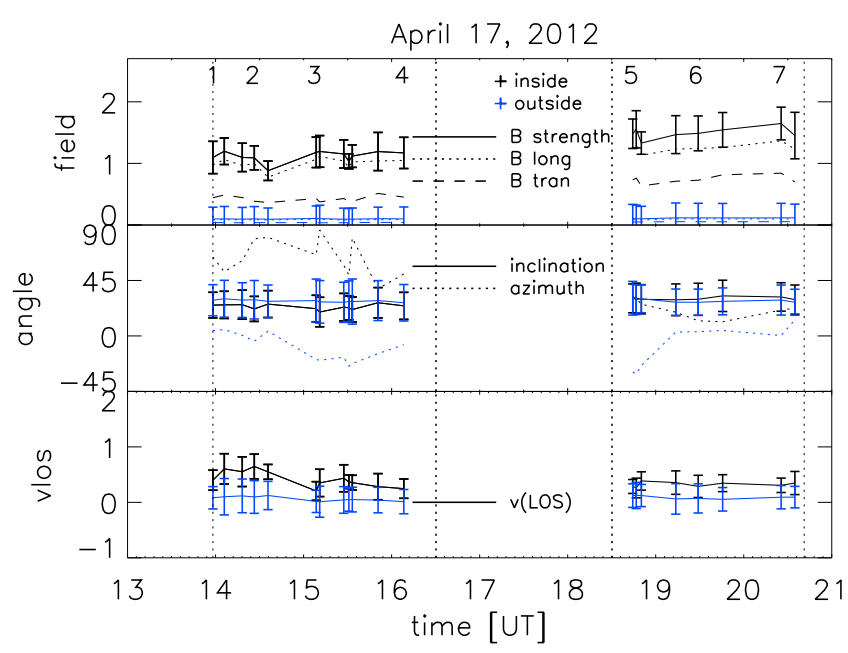

Fig. 7. Variation of the magnetic field strength ( $B$ strength), transverse ( $B$ tran), and longitudinal ( $B$ long) components in the LSF (top panel), field inclination, and azimuth in the LSF (middle panel), and LOS plasma velocity $v_{\text {LOS }}$ (bottom panel) derived from the IBIS photospheric Fe I $617.3 \mathrm{~nm}$ line data during the pore formation, inside (black lines) and outside the evolving feature (blue lines). Each plot shows the mean and standard deviation of the values derived from the data inversion and other methods described in Sect. 2. Field, angle, and velocity values are given in $\mathrm{kG}$, degree, and $\mathrm{km} \mathrm{s}^{-1}$ units, respectively. For the sake of clarity, the standard deviation is shown only for magnetic field strength, inclination, and $v_{\text {LOS }}$ estimates, inside and around the evolving structure. The vertical dotted lines show the time interval lacking IBIS observations. The numbers in the upper panel indicate the seven times corresponding to the evolutionary stages shown in Figs. 5 and 6.

in previous stages (Fig. 7, bottom panel); outside the evolving pore, the velocity of the plasma motions does not change significantly over the analysed period.

\section{Discussion and conclusions}

The results derived from our analysis agree with the outcomes of former studies of steady pores mentioned above; specifically, with the magnetic field patterns and strengths presented, for example by Sobotka et al. (2012), and the $v_{\mathrm{LOS}}$ and $v_{\mathrm{H}}$ reported by Cho et al. (2010), Sobotka et al. (2012, 2013), and Verma et al. (2016), for example. However, unlike previous studies, our data also allow us to investigate the properties of the photospheric plasma in the evolving region during the pore formation, and thus provide further observational constraints to numerical models of the AR evolution.

Magnetic fields and fluid motions are coupled according to the equations of the magnetohydrodynamics in 3D space and time. Cheung et al. (2008) performed simulations to investigate the interaction between convection and a magnetic flux tube rising into the photosphere, and discussed the outcomes with respect to observations from the HINODE mission. They reported that the rising flux tube expands because of the strong stratification of the convective zone by forming a magnetic sheet that acts as a reservoir for small-scale flux emergence events occurring at the scale of granulation. They also found that the interaction of the convective downflows and the rising magnetic flux tube undulates it to form serpentine field lines that emerge into the photosphere.

Later on, from an in-depth progression of the above simulations to the formation of an AR, Cheung et al. (2010) showed that the above serpentine fields gradually coalesce to form larger 
magnetic concentrations that eventually form a pair of opposite polarity spots. They also pointed out that correlations between the magnetic field and velocity field fluctuations allow the spots to accumulate flux by Lorentz-force-driven, counter streaming motion of opposite polarity regions.

From recent 3D MHD investigations of the effects of inflows in the evolution of ARs, Rempel \& Cheung (2014) reported that, in their simulated photosphere, the flux appears organized on a granular scale with mostly mixed polarity with magnetic flux of the order $10^{21} \mathrm{Mx}$ and average value lower than $0.1 \mathrm{kG}$. After emergence, the simulated dipoles undergo horizontal diverging flows, reaching an amplitude of up to $2 \mathrm{~km} \mathrm{~s}^{-1}$. In the numerical domain, these motions produce a progressive separation of the polarity of the dipoles that migrate in the opposite direction, by moving the opposite polarity flux away from the emergence regions.

The above simulations have contributed to a long series of numerical studies aimed at the identification of the mechanisms responsible for the formation and evolution of solar magnetic structures (see e.g. Cameron et al. 2007, 2011; Cheung et al. 2008, 2010; Fang et al. 2012b,a, 2014; Kitiashvili et al. 2010; Martinez-Sykora 2012; Martínez-Sykora et al. 2015; Stein et al. 2011; Stein \& Nordlund 2012; Toriumi \& Yokoyama 2012, 2013, to mention those that have been presented during the last decade). Although these studies can strongly differ in terms of the initial conditions and scales of the simulated processes, they have all reproduced some flux evolution signatures in agreement with observations. Hence the question arises on which processes unveiled by the numerical studies can be considered robust with both the observations and model assumptions.

In this regard, the data analysed in our study show several observational facts that are consistent with the outcomes of the MHD simulations presented by Rempel \& Cheung (2014). In particular, we found as follows:

- At the initial stages of the AR evolution, the analysed observations display mixed polarity flux patches organized on a granular scale; the flux patches in the trailing region of the forming AR are more clearly seen than those in the leading part (e.g. Fig. 1 and attached movie).

- After about $24 \mathrm{~h}$, the patches in the leading part become stronger than those in the trailing region and form a filamentary, sheet-like, coherent structure (e.g. movie available online and Figs. 1-3); these are the observed initial stages of pore formation.

- At that time, the small-scale mixed polarity patches hold flux of the order $10^{21} \mathrm{Mx}$ in an evolving region with an average field below $0.1 \mathrm{kG}$ (e.g. Figs. 1, 2, 5).

- The flux then shows an increase of comparable magnitude in both polarity patches of the evolving region (e.g. Fig. 2).

- At a later time, the data clearly display small-scale opposite polarity features that counterstream, coalesce, and reinforce flux accumulation at distinct sites (e.g. movie available online and Fig. 4).

- At that time, elongated granules appear in close proximity of the evolving feature, mostly located in the region the opposite polarities of the evolving AR facing each other (e.g. Fig. 3).

- The pore increases in size, while the sites of flux accumulation move away from each other with clear horizontal diverging motions and a rather small increase of the average field in the forming pore (e.g. Figs. 4-6).

- Horizontal diverging motions seem to produce further aggregation of field of the same polarity (e.g. movies available online and Figs. 4, 6); the plasma velocity is up to $0.4 \mathrm{~km} \mathrm{~s}^{-1}$ in the forming pore and up to $1 \mathrm{~km} \mathrm{~s}^{-1}$ outside it.

- Strong downflows, with plasma velocity $>1.5 \mathrm{~km} \mathrm{~s}^{-1}$, appear near the periphery of the forming pore and where magnetic flux accumulates (e.g. Fig. 6).

- Most intense field concentrations occur near the edges of the magnetic regions in evolution (e.g. Figs. 5, 6) as due to the confinement of the field by the ambient plasma motions.

- The analysed data show that the pore formation in the leading region of the AR occurs rapidly $(<1 \mathrm{~h})$; the evolution of the flux patch in the leading part is faster $(<12 \mathrm{~h})$ than the evolution (20-30 h) of the more diffuse and smaller scale flux patches in the trailing region (e.g. supplemental movies and Fig. 1).

- At the final stages of the AR evolution, about $48 \mathrm{~h}$ after the pore formation, the evolution of the region leads to the formation of a large-scale AR with a magnetic flux of the order of $10^{22} \mathrm{Mx}$.

From analysis of two relatively isolated ARs observed from the SDO mission, Centeno (2012) already reported some observational signatures of AR formation consistent with the 3D MHD numerical simulation of a rising-tube process, such as evidence of a connection between horizontal field patches and strong upflows, elongated granulation around the evolving ARs, and a mass discharge process through magnetic reconnection, as envisaged in the simulations of Cheung et al. (2010) and Rempel \& Cheung (2014), for example. In contrast, from a recent study of HINODE spectro-polarimetric observations of a young dipolar subregion developing within an AR, Getling et al. (2016) presented observational results that are considered to conflict with the signatures expected by the emergence of a flux-tube loop. In particular, they reported a fountain-like 3D magnetic structure of the studied features and lack of large-scale (horizontal and vertical) flows over the evolving area, which are seen as two pieces of evidence of magnetic region generation by local convective dynamo mechanisms as envisaged in the 3D MHD simulations, for example by Stein \& Nordlund (2012).

Compared to the HINODE and SDO observations analysed, for example by Getling et al. (2016) and Centeno (2012), the IBIS data considered in our study have spatial and temporal resolution that are higher at least by factors 3.5 and 11, respectively. By analysing the available data, we found that the simulations of the rising-tube process successfully reproduce both the average properties of the physical quantities estimated in the studied region and the mechanisms driving the observed pore formation. In particular, the studied pore seems to result from the emergence into the photosphere of a strong field formed in the solar interior, with some amplification and structuring effects of the initially emerged field by surface plasma motions, as evinced from the simulations for example by Rempel \& Cheung (2014). The above simulations also describe the evolution of the studied region at different spatial and temporal scales fairly well. The signatures observed in the studied region, in contrast, differ from those presented by Getling et al. (2016), which support the scenario of pore and larger scale magnetic region generation by local convective dynamo mechanisms.

Acknowledgements. The authors wish to thank Serena Criscuoli, Han Uitenbroek, and the whole DST staff for its support during the observing campaign and data reduction. This study received funding from the European Unions Seventh Programme for Research, Technological Development and Demonstration, under the Grant Agreements of the eHEROES (No. 284461, www. eheroes.eu), SOLARNET (No. 312495, www. solarnet-east.eu), and SOLID (No. 313188, projects.pmodwrc.ch/solid/) projects. This work was also supported by the Istituto Nazionale di Astrofisica (PRIN-INAF-2014) 
and Italian MIUR (PRIN-2012). We thank the anonymous referee for useful comments and suggestions. The National Solar Observatory is operated by the Association of Universities for Research in Astronomy under a cooperative agreement with the National Science Foundation.

\section{References}

Asensio Ramos, A., Manso Sainz, R., Martínez González, M. J., et al. 2012, ApJ, 748,83

Balthasar, H., Collados, M., \& Muglach, K. 2000, Astron. Nachr., 321, 121

Bellot Rubio, L. R., Ruiz Cobo, B., \& Collados, M. 2000, ApJ, 535, 489

Bellot Rubio, L. R. 2003, in Solar Polarization, eds. J. Trujillo-Bueno, \& J. Sanchez Almeida, ASP Conf. Ser., 307, 301

Bello González, N., Kneer, F., \& Schlichenmaier, R. 2012, A\&A, 538, A62

Bobra, M. G., Sun, X., Hoeksema, J. T., et al. 2014, Sol. Phys., 289, 3549

Bogdan, T. J., \& Judge, P. G. 2006, Roy. Soc. London Phil. Trans. Ser. A, 364, 313

Buehler, D., Lagg, A., van Noort, M., \& Solanki, S. K. 2016, A\&A, 589, A31

Cameron, R., Schüssler, M., Vögler, A., \& Zakharov, V. 2007, A\&A, 474, 261

Cameron, R., Vögler, A., \& Schüssler, M. 2011, A\&A, 533, A86

Cavallini, F. 2006, Sol. Phys., 236, 415

Centeno, R. 2012, ApJ, 759, 72

Cheung, M. C. M., Schüssler, M., Tarbell, T. D., \& Title, A. M. 2008, ApJ, 687, 1373

Cheung, M. C. M., Rempel, M., Title, A. M., \& Schüssler, M. 2010, ApJ, 720, 233

Cho, K.-S., Bong, S.-C., Chae, J., Kim, Y.-H., \& Park, Y.-D. 2010, ApJ, 723, 440

Cho, K.-S., Bong, S.-C., Chae, J., et al. 2013, Sol. Phys., 288, 23

Ermolli, I., Giorgi, F., Romano, P., et al. 2014, Sol. Phys., 289, 2525

Fang, F., Manchester, IV, W., Abbett, W. P., \& van der Holst, B. 2012a, ApJ, 754 15

Fang, F., Manchester, IV, W., Abbett, W. P., \& van der Holst, B. 2012b, ApJ, 745, 37

Fang, F., Fan, Y., \& McIntosh, S. W. 2014, ApJ, 789, L19

Fisher, G. H., \& Welsch, B. T. 2008, in Subsurface and Atmospheric Influences on Solar Activity, eds. R. Howe, R. W. Komm, K. S. Balasubramaniam, \& G. J. D. Petrie, ASP Conf. Ser., 383, 373

Georgoulis, M. K. 2005, ApJ, 629, L69

Getling, A. V., Ishikawa, R., \& Buchnev, A. A. 2016, Sol. Phys., 291, 371

Gingerich, O., Noyes, R. W., Kalkofen, W., \& Cuny, Y. 1971, Sol. Phys., 18, 347

Giordano, S., Berrilli, F., Del Moro, D., \& Penza, V. 2008, A\&A, 489, 747

Guglielmino, S. L., \& Zuccarello, F. 2011, ApJ, 743, L9

Hapgood, M. 2012, Nature, 484, 311

Hoeksema, J. T., Liu, Y., Hayashi, K., et al. 2014, Sol. Phys., 289, 3483

Kitiashvili, I. N., Kosovichev, A. G., Wray, A. A., \& Mansour, N. N. 2010, ApJ, 719,307
Lagg, A., Woch, J., Solanki, S. K., \& Krupp, N. 2007, A\&A, 462, 1147 Martinez-Sykora, J. 2012, IAU Special Session, 6, 105

Martínez-Sykora, J., Moreno-Insertis, F., \& Cheung, M. C. M. 2015, ApJ, 814, 2 Norton, A. A., Graham, J. P., Ulrich, R. K., et al. 2006, Sol. Phys., 239, 69 Pesnell, W. D., Thompson, B. J., \& Chamberlin, P. C. 2012, Sol. Phys., 275, 3 Reardon, K. P., \& Cavallini, F. 2008, A\&A, 481, 897

Rempel, M., \& Cheung, M. C. M. 2014, ApJ, 785, 90

Rempel, M., \& Schlichenmaier, R. 2011, Liv. Rev. Sol. Phys., 8, 3

Requerey, I. S., Del Toro Iniesta, J. C., Bellot Rubio, L. R., et al. 2015, ApJ, 810, 79

Rezaei, R., Bello González, N., \& Schlichenmaier, R. 2012, A\&A, 537, A19

Rimmele, T. R., Richards, K., Hegwer, S., et al. 2004, in Telescopes and Instrumentation for Solar Astrophysics, eds. S. Fineschi, \& M. A. Gummin, SPIE Conf. Ser., 5171, 179

Romano, P., Frasca, D., Guglielmino, S. L., et al. 2013, ApJ, 771, L3

Romano, P., Guglielmino, S. L., Cristaldi, A., et al. 2014, ApJ, 784, 10

Ruiz Cobo, B., \& del Toro Iniesta, J. C. 1992, ApJ, 398, 375

Sainz Dalda, A., Vargas Domínguez, S., \& Tarbell, T. D. 2012, ApJ, 746, L13

Scherrer, P. H., Schou, J., Bush, R. I., et al. 2012, Sol. Phys., 275, 207

Schlichenmaier, R., Rezaei, R., Bello González, N., \& Waldmann, T. A. 2010, A\&A, 512, L1

Schou, J., Scherrer, P. H., Bush, R. I., et al. 2012, Sol. Phys., 275, 229

Schuck, P. W. 2006, ApJ, 646, 1358

Schuck, P. W. 2008, ApJ, 683, 1134

Sobotka, M. 2003, Astron. Nachr., 324, 369

Sobotka, M., Del Moro, D., Jurčák, J., \& Berrilli, F. 2012, A\&A, 537, A85

Sobotka, M., Švanda, M., Jurčák, J., et al. 2013, A\&A, 560, A84

Solanki, S. K. 2003, A\&ARv, 11, 153

Stangalini, M., Del Moro, D., Berrilli, F., \& Jefferies, S. M. 2011, A\&A, 534, A65

Stangalini, M., Giannattasio, F., Del Moro, D., \& Berrilli, F. 2012, A\&A, 539, L4

Stein, R. F., \& Nordlund, Å. 2012, ApJ, 753, L13

Stein, R. F., Lagerfjärd, A., Nordlund, A., \& Georgobiani, D. 2011, Sol. Phys., 268, 271

Toriumi, S., \& Yokoyama, T. 2012, A\&A, 539, A22

Toriumi, S., \& Yokoyama, T. 2013, A\&A, 553, A55

Toriumi, S., Hayashi, K., \& Yokoyama, T. 2014, ApJ, 794, 19

van Noort, M., Rouppe van der Voort, L., \& Löfdahl, M. G. 2005, Sol. Phys., 228, 191

Vargas Domínguez, S., de Vicente, A., Bonet, J. A., \& Martínez Pillet, V. 2010, A\&A, 516, A91

Vargas Domínguez, S., MacTaggart, D., Green, L., van Driel-Gesztelyi, L., \& Hood, A. W. 2012, Sol. Phys., 278, 33

Verma, M., \& Denker, C. 2014, A\&A, 563, A112

Verma, M., Denker, C., Balthasar, H., et al. 2016, A\&A, 596, A3

Watanabe, H., Kitai, R., \& Otsuji, K. 2014, ApJ, 796, 77

Yang, G., Xu, Y., Wang, H., \& Denker, C. 2003, ApJ, 597, 1190 
Appendix A: Examples data inversion results
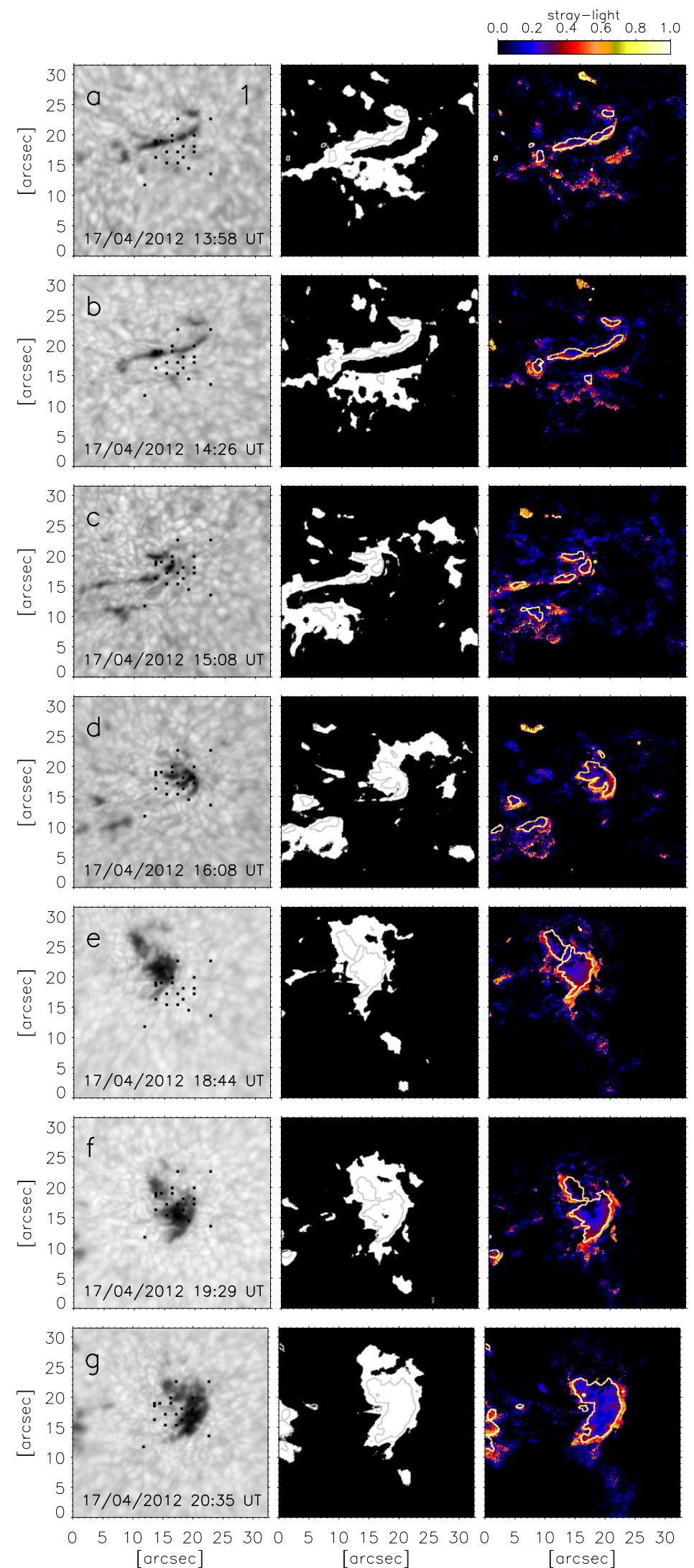

Fig. A.1. Example of continuum intensity images (left column), mask of the magnetic regions identified in the data (middle column, white (black) shows the magnetic (quiet) component) and stray-light fraction (right column) derived from the SIR inversion of the IBIS Fe I $617.3 \mathrm{~nm}$ line data, at seven stages of the pore formation at a) 13:58 UT, b) 14:26 UT, c) 15:08 UT, d) 16:08 UT, e) 18:44 UT, f) 19:29 UT, and g) 20:35 UT. North is at the top; west is to the right. The small black squares in the continuum intensity images show the 20 positions considered for the comparison between observed and inverted profiles in Figs. A.2-A.8. The contour line in the panels of the middle and right columns shows the location of the evolving structure singled out in the continuum data, as specified in Fig. 3. 

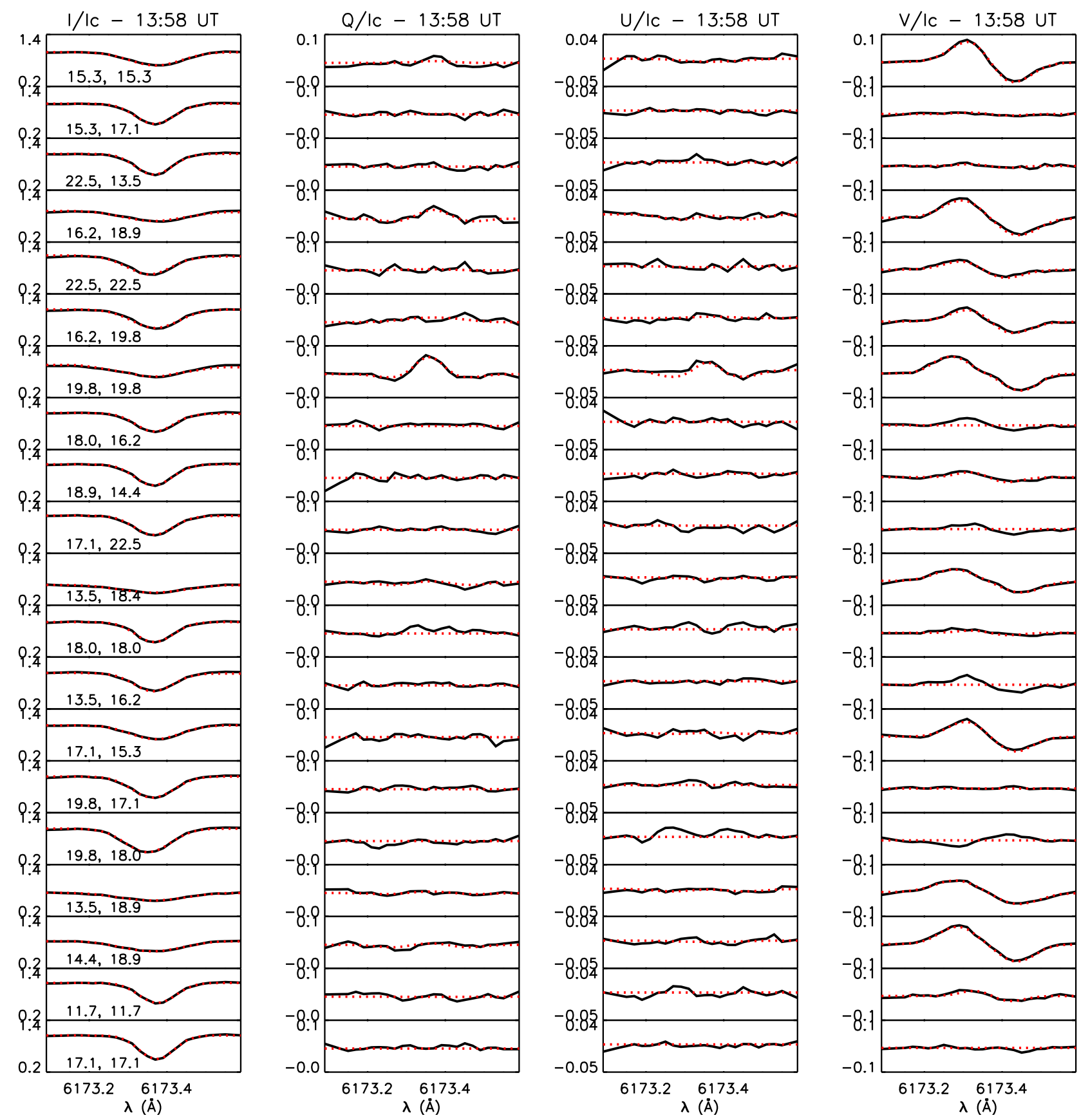

Fig. A.2. From left to right: examples of observed (black) and inverted (red) Stokes- $I,-Q,-U$, and $-V$ profiles at the 20 positions on the sub-FOV labelled (a) in Fig. A.1, taken during the pore formation at 13:58 UT. Spectra are normalized to the continuum intensity. Numbers on the bottom left in each sub-panel of the Stokes-I figure indicate the position of the analysed pixel expressed in arcsec with respect to the sub-FOV shown in Fig. A.1. Values of the stray-light fraction, LOS magnetic field strength $(\mathrm{kG})$, field inclination and azimuth (degree), and LOS velocity $\left(\mathrm{km} \mathrm{s}^{-1}\right)$ derived from our data analysis (at optical depth $\log \tau_{500}=1$ ) at the same positions of the shown profiles are listed in Table A.1; the values are cut at 2 decimal digits. 
I. Ermolli et al.: Velocity and magnetic fields in a forming pore

Table A.1. Summary of the plasma properties estimated at the 20 positions considered in Figs. A.2.

\begin{tabular}{rrrrrrr}
\hline \hline $\begin{array}{r}\text { Position } \\
x\end{array}$ & $\begin{array}{r}\text { Position } \\
y\end{array}$ & $\begin{array}{r}\text { Stray-light } \\
\text { fraction }\end{array}$ & $\begin{array}{r}\text { Field } \\
\text { strength } \\
(\mathrm{kG})\end{array}$ & $\begin{array}{r}\text { Field } \\
\text { arcseclination } \\
(\text { degree })\end{array}$ & $\begin{array}{r}\text { Field } \\
\text { azimuth } \\
(\text { degree })\end{array}$ & $\begin{array}{c}v_{\text {LOS }} \\
\left(\mathrm{km} \mathrm{s}^{-1}\right)\end{array}$ \\
\hline 15.3 & 15.3 & 0.00 & 0.67 & 25.33 & -22.11 & 0.33 \\
15.3 & 17.1 & 0.00 & 0.02 & 20.33 & -63.90 & 0.08 \\
22.5 & 13.5 & 0.00 & 0.03 & 19.88 & -86.65 & 0.11 \\
16.2 & 18.9 & 0.18 & 1.10 & 11.15 & 161.89 & 0.46 \\
22.5 & 22.5 & 0.17 & 0.12 & 18.45 & -87.51 & -0.11 \\
16.2 & 19.8 & 0.00 & 0.41 & 23.97 & 20.41 & 0.17 \\
19.8 & 19.8 & 0.38 & 1.15 & 18.99 & 150.45 & 0.22 \\
18.0 & 16.2 & 0.16 & 0.11 & 24.10 & -86.22 & 0.33 \\
18.9 & 14.4 & 0.00 & 0.07 & 19.68 & -90.49 & 0.01 \\
17.1 & 22.5 & 0.05 & 0.06 & 16.13 & 106.53 & 0.16 \\
13.5 & 18.4 & 0.01 & 1.19 & 19.66 & 100.73 & 0.24 \\
18.0 & 18.0 & 0.00 & 0.07 & 35.82 & -71.08 & 0.19 \\
13.5 & 16.2 & 0.11 & 0.18 & 18.92 & 55.18 & 0.13 \\
17.1 & 15.3 & 0.00 & 0.52 & 28.09 & -69.31 & 0.34 \\
19.8 & 17.1 & 0.00 & 0.02 & 47.26 & -100.35 & 0.01 \\
19.8 & 18.0 & 0.00 & 0.00 & 76.06 & -105.08 & -0.37 \\
13.5 & 18.9 & 0.00 & 0.84 & 36.02 & -54.81 & 0.38 \\
14.4 & 18.9 & 0.00 & 0.82 & 34.25 & 17.01 & 0.16 \\
11.7 & 11.7 & 0.00 & 0.06 & 20.02 & 83.90 & 0.28 \\
17.1 & 17.1 & 0.00 & 0.02 & 25.53 & -80.95 & 0.18 \\
\hline
\end{tabular}

Table A.2. Summary of the plasma properties estimated at the 20 positions considered in Figs. A.3.

\begin{tabular}{rrrrrrr}
\hline \hline $\begin{array}{r}\text { Position } \\
x\end{array}$ & $\begin{array}{r}\text { Position } \\
(\operatorname{arcsec})\end{array}$ & $\begin{array}{r}\text { Stray-light } \\
\text { fraction }\end{array}$ & $\begin{array}{r}\text { Field } \\
\text { strength } \\
(\mathrm{kG})\end{array}$ & $\begin{array}{r}\text { Field } \\
\text { inclination } \\
(\text { degree })\end{array}$ & $\begin{array}{r}\text { Field } \\
\text { azimuth } \\
(\text { degree })\end{array}$ & $v_{\text {LOS }}$ \\
$\left(\mathrm{km} \mathrm{s}^{-1}\right)$
\end{tabular}



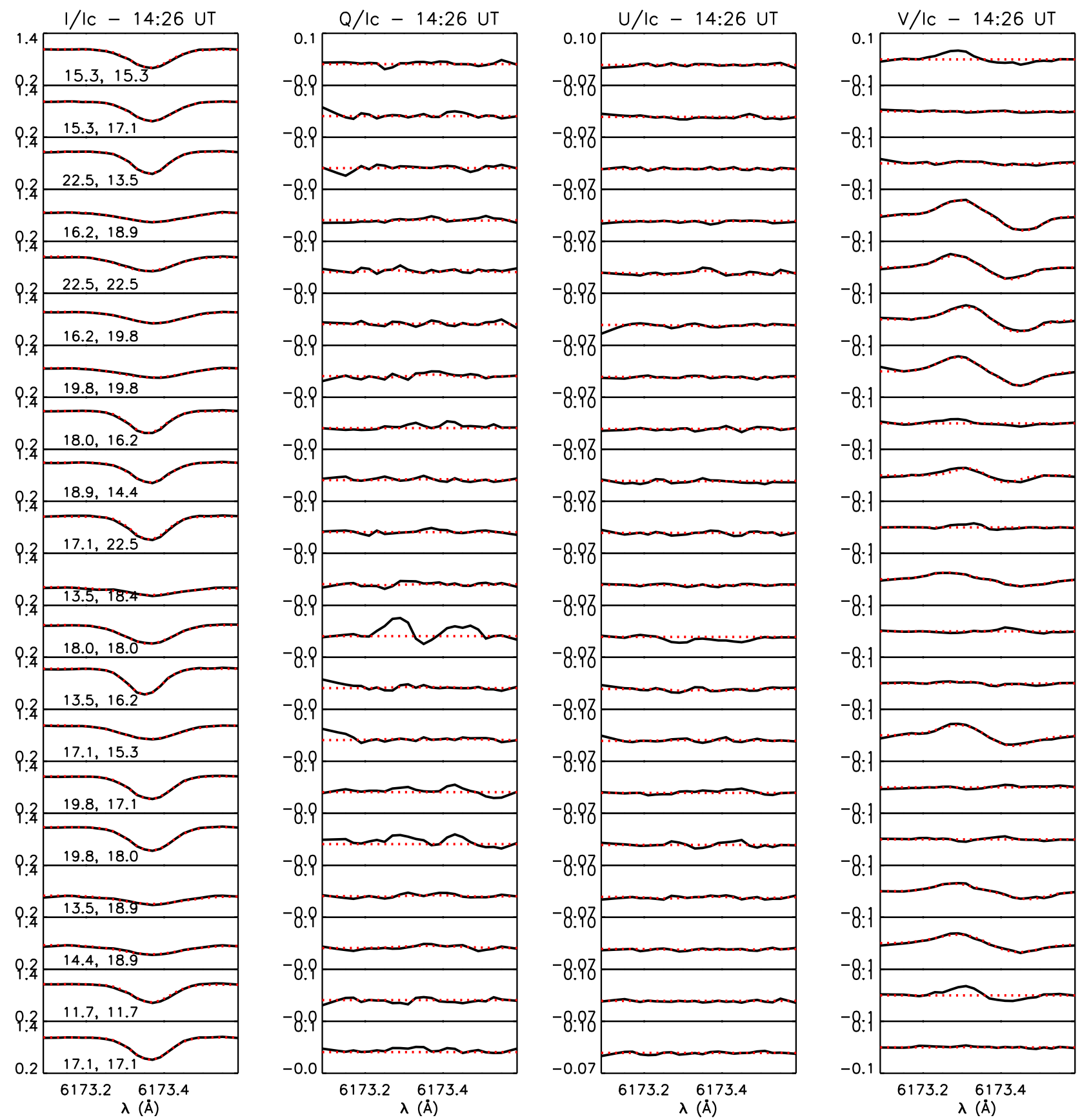

Fig. A.3. As in Fig. A.2 but for the data taken at 14:26 UT (stage (b) of the pore formation shown in Fig. A.1). Values of the stray-light fraction, LOS magnetic field strength $(\mathrm{kG})$, field inclination and azimuth (degree), and LOS velocity $\left(\mathrm{km} \mathrm{s}^{-1}\right)$ derived from our data analysis (at optical depth $\left.\log \tau_{500}=1\right)$ at the same positions of the shown profiles are listed in Table A.2; the values are cut at 2 decimal digits. 
I. Ermolli et al.: Velocity and magnetic fields in a forming pore
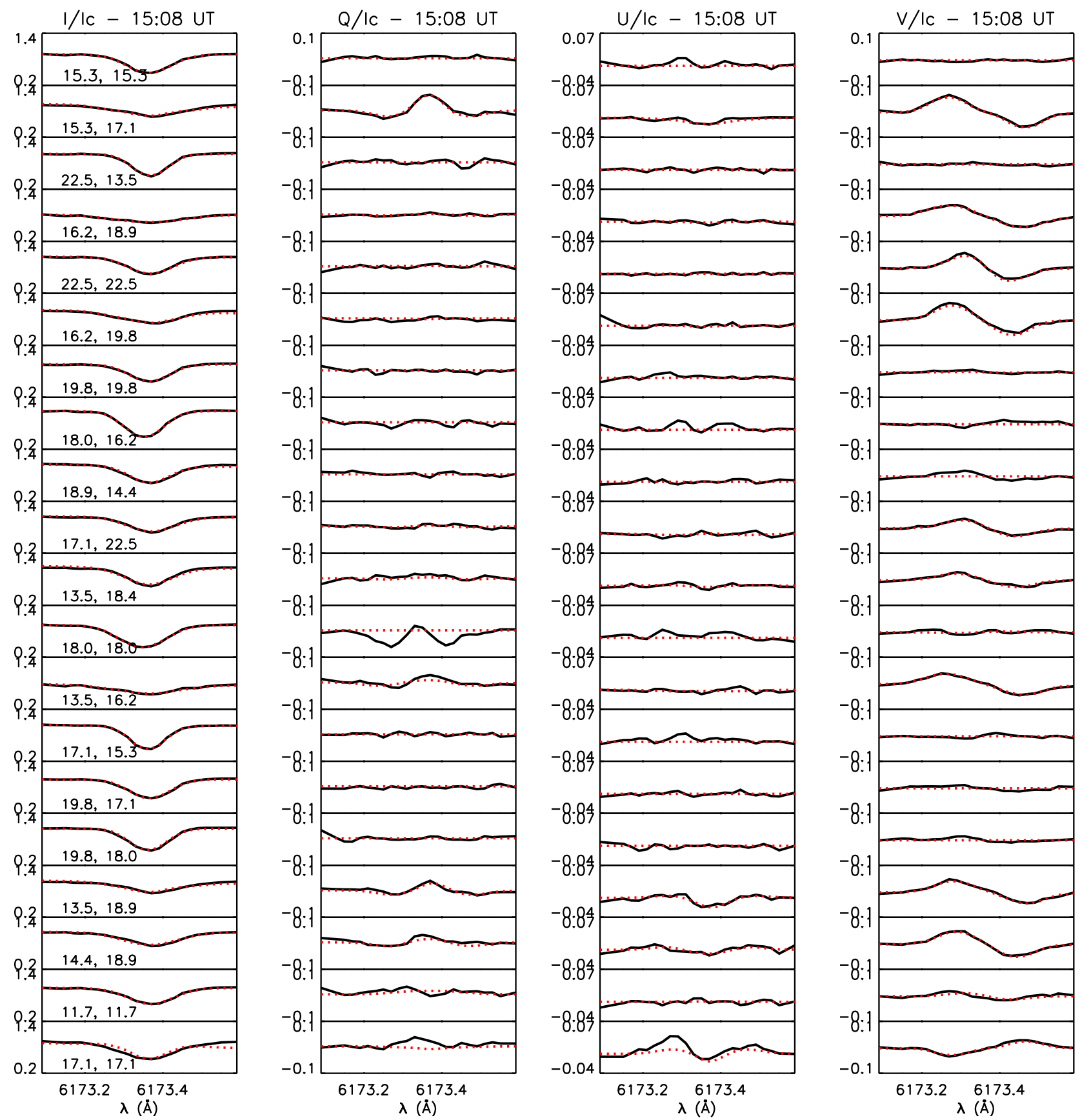

Fig. A.4. As in Fig. A.2 but for the data taken at 15:08 UT (stage (c) of the pore formation shown in Fig. A.1). Values of the stray-light fraction, LOS magnetic field strength $(\mathrm{kG})$, field inclination and azimuth (degree), and LOS velocity $\left(\mathrm{km} \mathrm{s}^{-1}\right)$ derived from our data analysis (at optical depth $\log \tau_{500}=1$ ) at the same positions of the shown profiles are listed in Table A.3; the values are cut at 2 decimal digits. 
Table A.3. Summary of the plasma properties estimated at the 20 positions considered in Figs. A.4.

\begin{tabular}{rrrrrrr}
\hline \hline $\begin{array}{r}\text { Position } \\
x\end{array}$ & $\begin{array}{r}\text { Position } \\
y\end{array}$ & $\begin{array}{r}\text { Stray-light } \\
\text { fraction }\end{array}$ & $\begin{array}{r}\text { Field } \\
\text { strength } \\
(\mathrm{kG})\end{array}$ & $\begin{array}{r}\text { Field } \\
\text { arcseclination } \\
(\text { degree })\end{array}$ & $\begin{array}{r}\text { Field } \\
\text { azimuth } \\
(\text { degree })\end{array}$ & $\begin{array}{c}v_{\text {LOS }} \\
\left(\mathrm{km} \mathrm{s}^{-1}\right)\end{array}$ \\
\hline 15.3 & 15.3 & 0.010 & 0.00 & 106.61 & -109.23 & 0.06 \\
15.3 & 17.1 & 0.37 & 1.30 & 17.66 & 49.68 & 0.37 \\
22.5 & 13.5 & 0.00 & 0.01 & 19.72 & -91.58 & 0.06 \\
16.2 & 18.9 & 0.00 & 1.24 & 26.84 & -69.30 & 0.25 \\
22.5 & 22.5 & 0.04 & 0.36 & 17.15 & 58.38 & 0.19 \\
16.2 & 19.8 & 0.47 & 0.84 & 17.13 & -110.95 & 0.12 \\
19.8 & 19.8 & 0.00 & 0.03 & 23.16 & -93.35 & 0.09 \\
18.0 & 16.2 & 0.00 & 0.02 & 115.11 & -124.39 & -0.28 \\
18.9 & 14.4 & 0.35 & 0.08 & 21.14 & -109.74 & 0.39 \\
17.1 & 22.5 & 0.14 & 0.32 & 17.75 & -95.71 & 0.27 \\
13.5 & 18.4 & 0.33 & 0.22 & 17.21 & 98.03 & -0.01 \\
18.0 & 18.0 & 0.00 & 0.00 & 83.34 & -158.86 & -0.56 \\
13.5 & 16.2 & 0.12 & 1.35 & 8.02 & 164.21 & 0.22 \\
17.1 & 15.3 & 0.10 & 0.03 & 127.02 & -108.06 & 0.07 \\
19.8 & 17.1 & 0.00 & 0.06 & 23.82 & -90.13 & 0.17 \\
19.8 & 18.0 & 0.00 & 0.03 & 19.57 & -99.72 & -0.08 \\
13.5 & 18.9 & 0.38 & 1.16 & 12.59 & 125.59 & 0.66 \\
14.4 & 18.9 & 0.10 & 0.97 & 7.91 & -0.98 & 0.34 \\
11.7 & 11.7 & 0.00 & 0.22 & 41.89 & -72.44 & 0.13 \\
17.1 & 17.1 & 0.75 & 0.20 & 62.52 & -122.73 & 0.09 \\
\hline
\end{tabular}

Table A.4. Summary of the plasma properties estimated at the 20 positions considered in Figs. A.5.

\begin{tabular}{rrrrrrr}
\hline \hline $\begin{array}{r}\text { Position } \\
x\end{array}$ & $\begin{array}{r}\text { Position } \\
y\end{array}$ & $\begin{array}{r}\text { Stray-light } \\
\text { fraction }\end{array}$ & $\begin{array}{r}\text { Field } \\
\text { strength } \\
(\mathrm{kG})\end{array}$ & $\begin{array}{r}\text { Field } \\
\text { inclination } \\
(\text { degree })\end{array}$ & $\begin{array}{r}\text { Field } \\
\text { azimuth } \\
(\text { degree })\end{array}$ & $v_{\text {LOS }}$ \\
$\left(\mathrm{km} \mathrm{s}^{-1}\right)$
\end{tabular}


I. Ermolli et al.: Velocity and magnetic fields in a forming pore
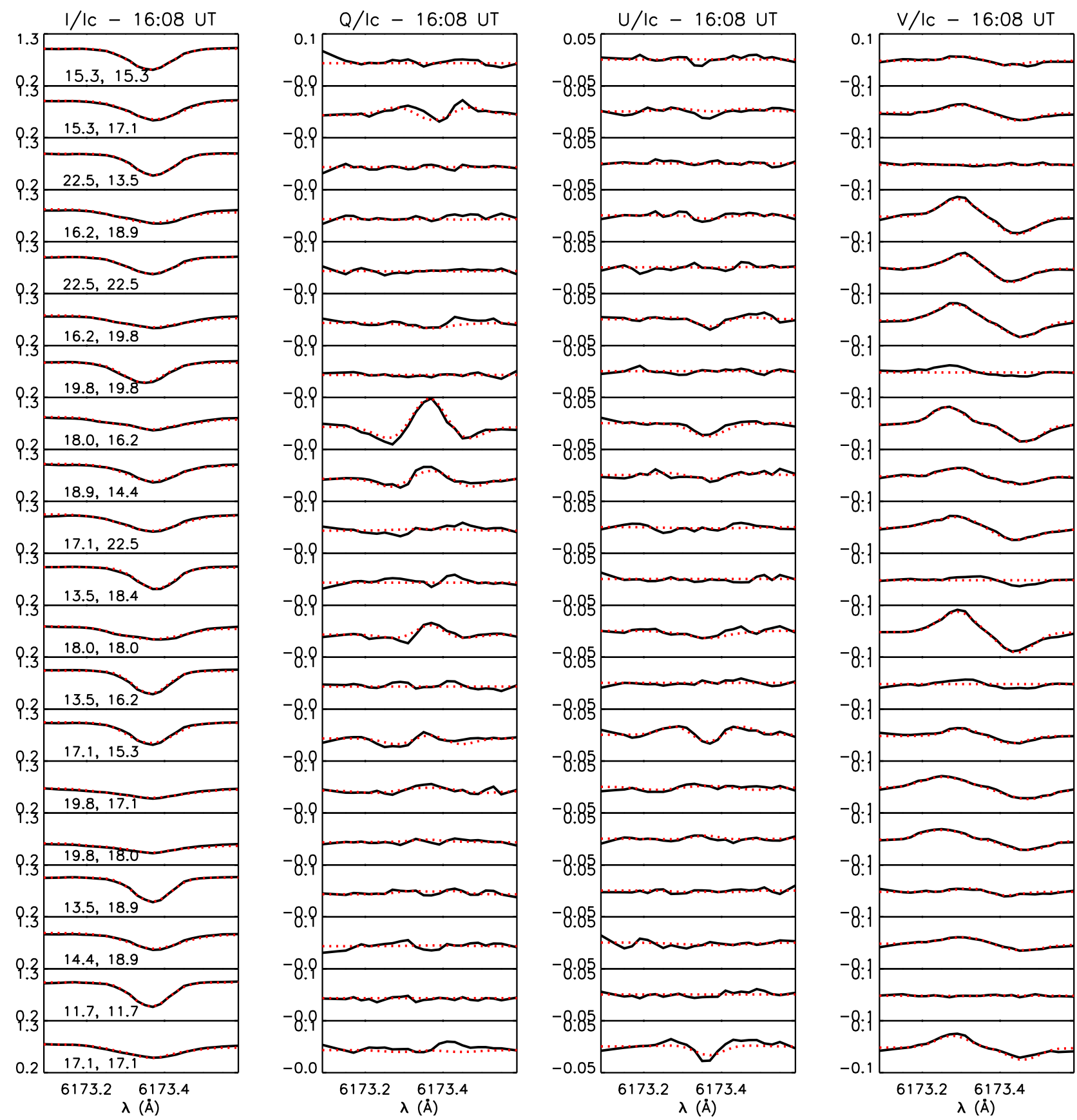

Fig. A.5. As in Fig. A.2 but for the data taken at 16:08 UT (stage (d) of the pore formation shown in Fig. A.1). Values of the stray-light fraction, LOS magnetic field strength $(\mathrm{kG})$, field inclination and azimuth (degree), and LOS velocity $\left(\mathrm{km} \mathrm{s}^{-1}\right)$ derived from our data analysis (at optical depth $\log \tau_{500}=1$ ) at the same positions of the shown profiles are listed in Table A.4; the values are cut at 2 decimal digits. 

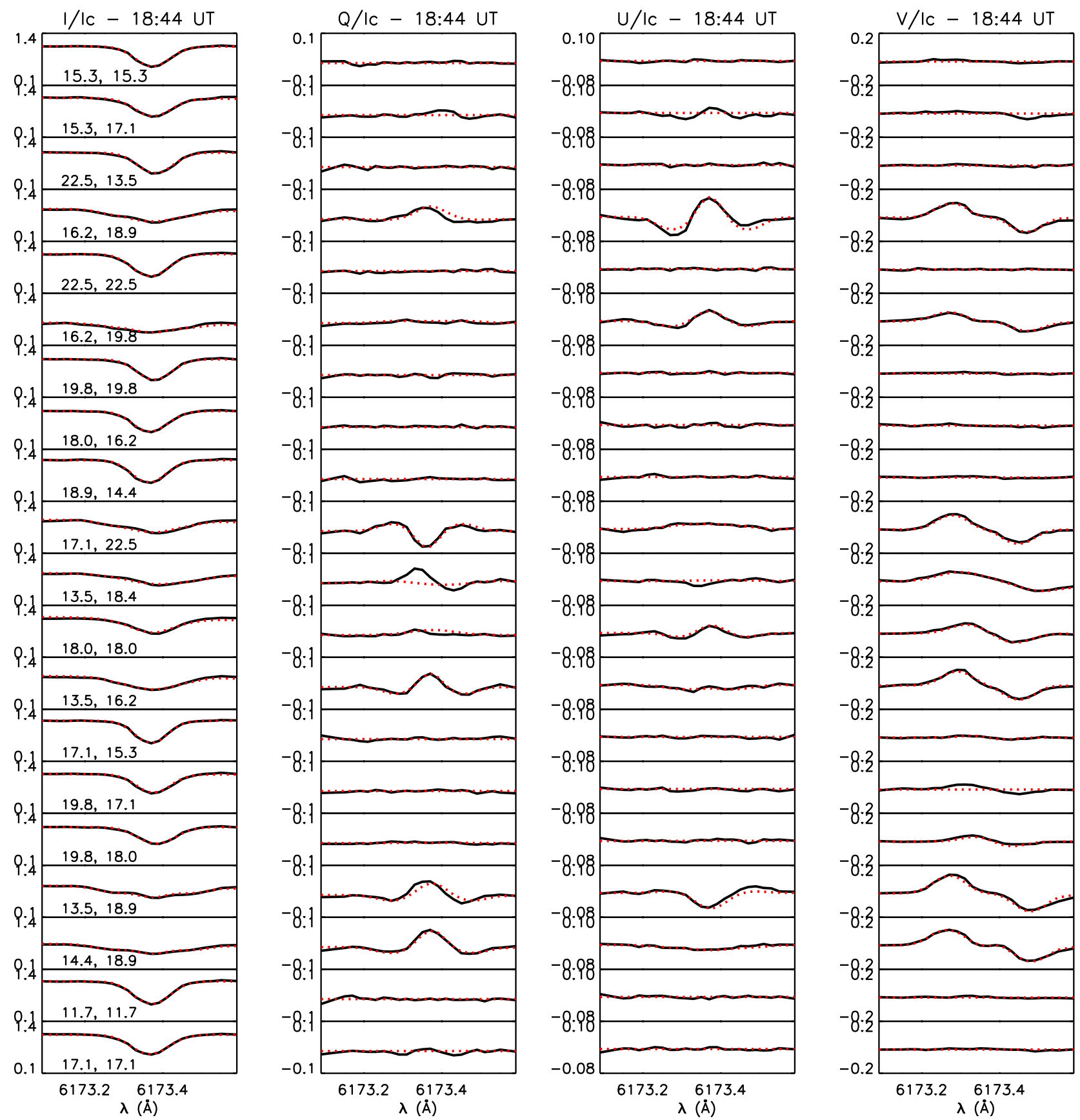

Fig. A.6. As in Fig. A.2 but for the data taken at 18:44 UT (stage (e) of the pore formation shown in Fig. A.1). Values of the stray-light fraction, LOS magnetic field strength $(\mathrm{kG})$, field inclination and azimuth (degree), and LOS velocity $\left(\mathrm{km} \mathrm{s}^{-1}\right)$ derived from our data analysis (at optical depth $\left.\log \tau_{500}=1\right)$ at the same positions of the shown profiles are listed in Table A.5; the values are cut at 2 decimal digits. 
I. Ermolli et al.: Velocity and magnetic fields in a forming pore

Table A.5. Summary of the plasma properties estimated at the 20 positions considered in Figs. A.6.

\begin{tabular}{rrrrrrr}
\hline \hline $\begin{array}{r}\text { Position } \\
x\end{array}$ & $\begin{array}{r}\text { Position } \\
y\end{array}$ & $\begin{array}{r}\text { Stray-light } \\
\text { fraction }\end{array}$ & $\begin{array}{r}\text { Field } \\
\text { strength } \\
(\mathrm{kG})\end{array}$ & $\begin{array}{r}\text { Field } \\
\text { inclination } \\
(\text { degree })\end{array}$ & $\begin{array}{r}\text { Field } \\
\text { azimuth } \\
(\text { degree })\end{array}$ & $\begin{array}{c}v_{\text {LOS }} \\
\left(\mathrm{km} \mathrm{s}^{-1}\right)\end{array}$ \\
\hline 15.3 & 15.3 & 0.05 & 0.03 & 20.64 & -86.64 & 0.34 \\
15.3 & 17.1 & 0.17 & 0.10 & 20.50 & 35.74 & 0.25 \\
22.5 & 13.5 & 0.00 & 0.01 & 27.04 & -89.94 & 0.25 \\
16.2 & 18.9 & 0.34 & 1.27 & 25.51 & 49.70 & 0.54 \\
22.5 & 22.5 & 0.00 & 0.01 & 22.83 & -91.06 & -0.04 \\
16.2 & 19.8 & 0.29 & 0.87 & 56.23 & -50.70 & 0.28 \\
19.8 & 19.8 & 0.00 & 0.02 & 19.52 & -92.04 & 0.23 \\
18.0 & 16.2 & 0.00 & 0.01 & 21.06 & -82.95 & 0.45 \\
18.9 & 14.4 & 0.00 & 0.02 & 21.55 & -81.66 & -0.20 \\
17.1 & 22.5 & 0.37 & 0.97 & 30.44 & -95.63 & 0.38 \\
13.5 & 18.4 & 0.10 & 1.13 & 19.32 & 125.05 & 1.17 \\
18.0 & 18.0 & 0.50 & 0.57 & 22.98 & 126.65 & 0.50 \\
13.5 & 16.2 & 0.37 & 0.96 & 17.62 & 155.54 & 0.31 \\
17.1 & 15.3 & 0.00 & 0.03 & 22.18 & -79.70 & 0.37 \\
19.8 & 17.1 & 0.10 & 0.08 & 25.69 & -68.98 & 0.30 \\
19.8 & 18.0 & 0.00 & 0.12 & 21.31 & -85.23 & 0.48 \\
13.5 & 18.9 & 0.36 & 1.50 & 14.60 & 112.04 & 0.63 \\
14.4 & 18.9 & 0.26 & 1.82 & 8.30 & 156.42 & 0.55 \\
11.7 & 11.7 & 0.00 & 0.03 & 26.85 & -103.30 & 0.00 \\
17.1 & 17.1 & 0.00 & 0.01 & 18.60 & -27.75 & 0.23 \\
\hline
\end{tabular}

Table A.6. Summary of the plasma properties estimated at the 20 positions considered in Figs. A.7.

\begin{tabular}{rrrrrrr}
\hline \hline $\begin{array}{r}\text { Position } \\
x\end{array}$ & $\begin{array}{r}\text { Position } \\
y\end{array}$ & $\begin{array}{r}\text { Stray-light } \\
\text { fraction }\end{array}$ & $\begin{array}{r}\text { Field } \\
\text { strength } \\
(\mathrm{kG})\end{array}$ & $\begin{array}{r}\text { Field } \\
\text { inclination } \\
(\text { degree })\end{array}$ & $\begin{array}{r}\text { Field } \\
\text { azimuth } \\
(\text { degree })\end{array}$ & $v_{\text {LOS }}$ \\
$\left(\mathrm{km} \mathrm{s}^{-1}\right)$
\end{tabular}



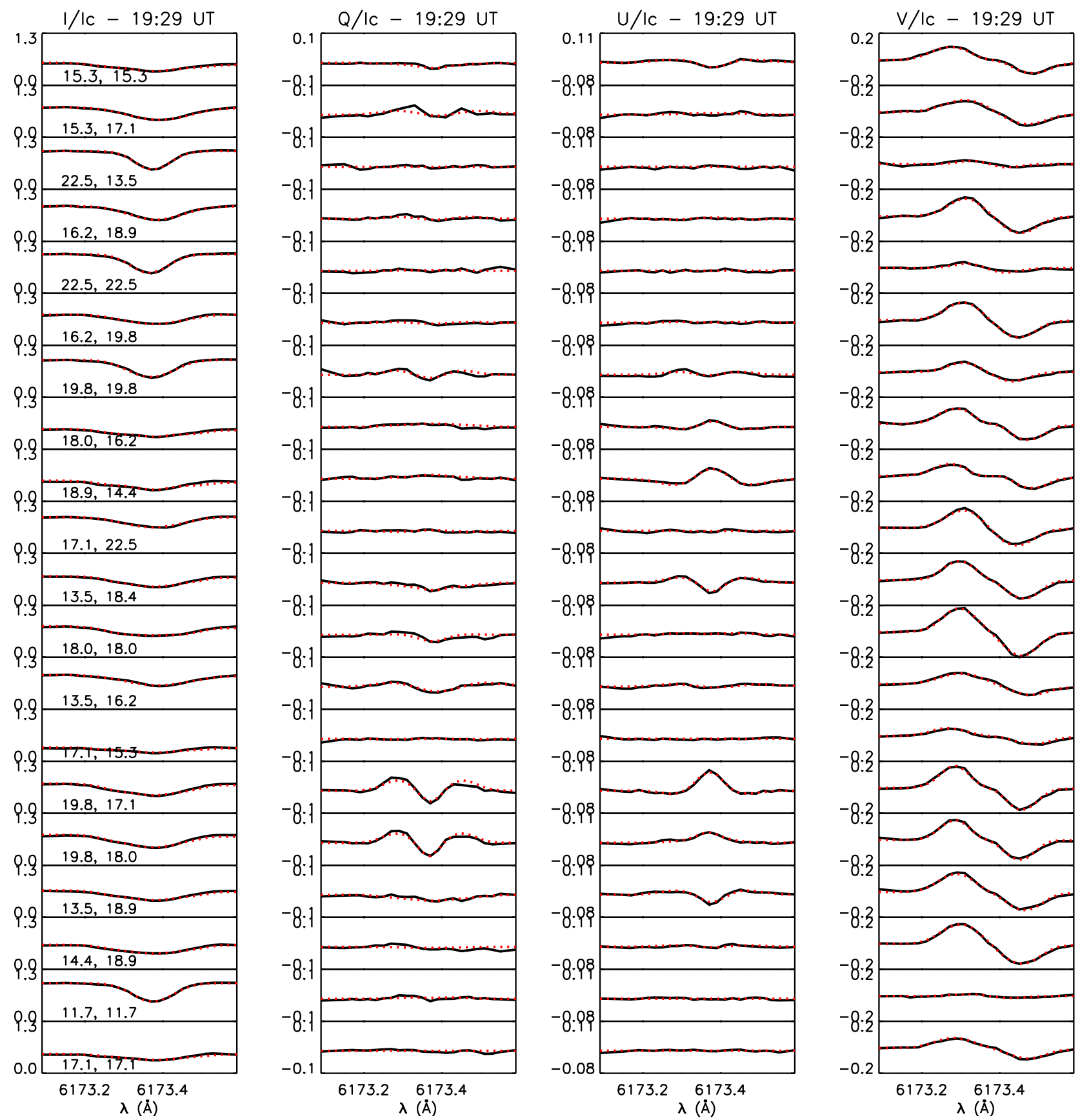

Fig. A.7. As in Fig. A.2 but for the data taken at 19:29 UT (stage (f) of the pore formation shown in Fig. A.1). Values of the stray-light fraction, LOS magnetic field strength $(\mathrm{kG})$, field inclination and azimuth (degree), and LOS velocity $\left(\mathrm{km} \mathrm{s}^{-1}\right)$ derived from our data analysis (at optical depth $\log \tau_{500}=1$ ) at the same positions of the shown profiles are listed in Table A.6; the values are cut at 2 decimal digits. 
I. Ermolli et al.: Velocity and magnetic fields in a forming pore
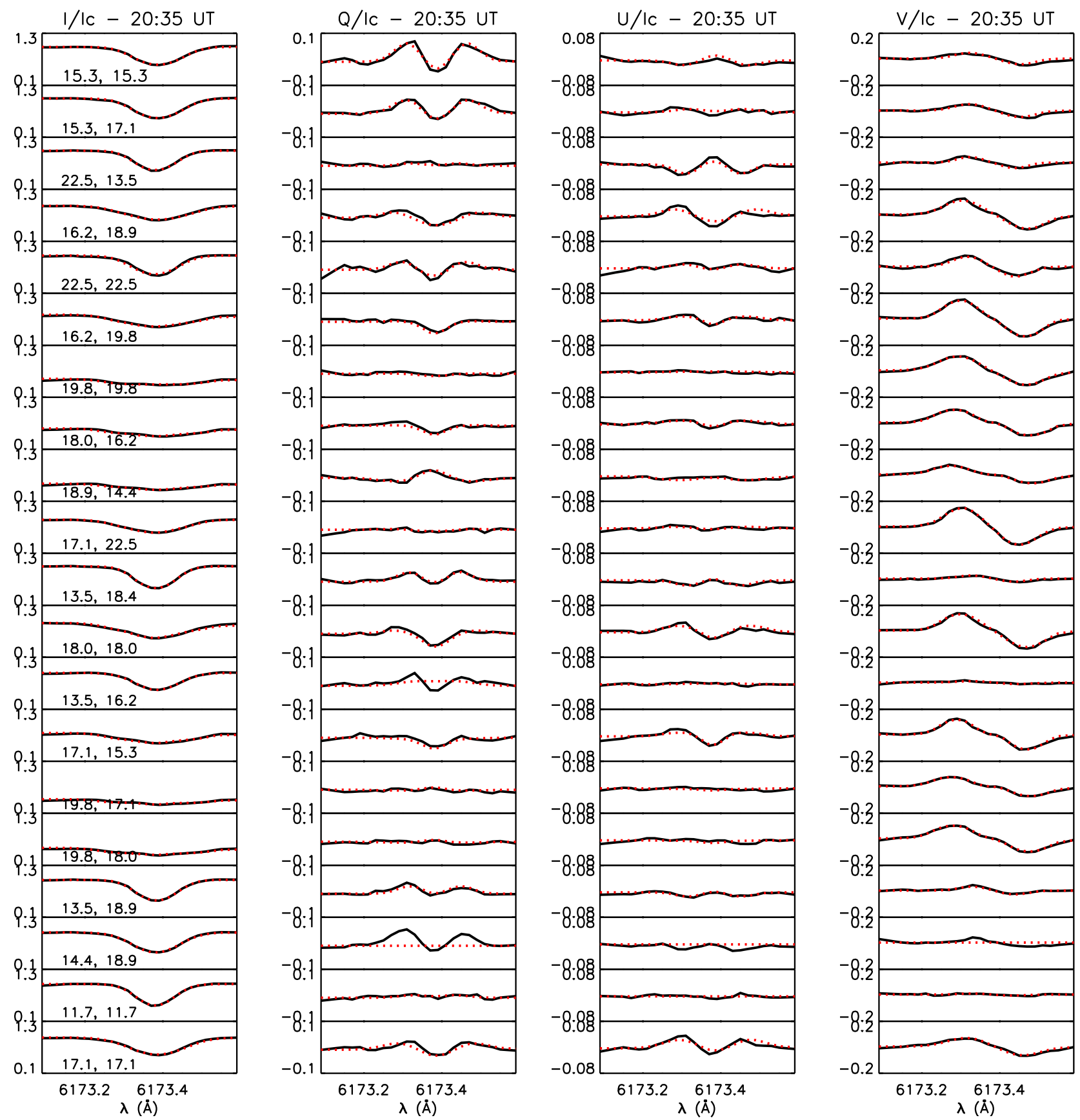

Fig. A.8. As in Fig. A.2 but for the data taken at 20:35 UT (stage (g) of the pore formation shown in Fig. A.1). Values of the stray-light fraction, LOS magnetic field strength $(\mathrm{kG})$, field inclination and azimuth (degree), and LOS velocity $\left(\mathrm{km} \mathrm{s}^{-1}\right)$ derived from our data analysis (at optical depth $\log \tau_{500}=1$ ) at the same positions of the shown profiles are listed in Table A.7; the values are cut at 2 decimal digits. 
A\&A 600, A102 (2017)

Table A.7. Summary of the plasma properties estimated at the 20 positions considered in Figs. A.8.

\begin{tabular}{rrrrrrr}
\hline \hline $\begin{array}{r}\text { Position } \\
x\end{array}$ & $\begin{array}{r}\text { Position } \\
y\end{array}$ & $\begin{array}{r}\text { Stray-light } \\
\text { fraction }\end{array}$ & $\begin{array}{r}\text { Field } \\
\text { strength } \\
(\mathrm{kG})\end{array}$ & $\begin{array}{r}\text { Field } \\
\text { inclination } \\
(\text { degree })\end{array}$ & $\begin{array}{r}\text { Field } \\
\text { azimuth } \\
(\text { degree })\end{array}$ & $v_{\text {LOS }}$ \\
$\left(\mathrm{km} \mathrm{s}^{-1}\right)$
\end{tabular}

